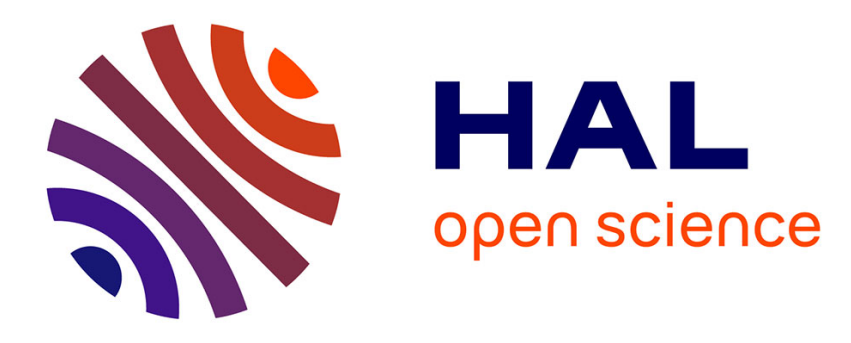

\title{
Mathematical and numerical study of a system of conservation laws
}

\author{
Robert Eymard, Elodie Tillier
}

\section{To cite this version:}

Robert Eymard, Elodie Tillier. Mathematical and numerical study of a system of conservation laws. Journal of Evolution Equations, 2007, http://www.springerlink.com/content/wrj46134231r8k66/?MUD=MP. 10.1007/s00028-006-0271$\mathrm{x}$. hal-00108526

\section{HAL Id: hal-00108526 https://hal.science/hal-00108526}

Submitted on 22 Oct 2006

HAL is a multi-disciplinary open access archive for the deposit and dissemination of scientific research documents, whether they are published or not. The documents may come from teaching and research institutions in France or abroad, or from public or private research centers.
L'archive ouverte pluridisciplinaire HAL, est destinée au dépôt et à la diffusion de documents scientifiques de niveau recherche, publiés ou non, émanant des établissements d'enseignement et de recherche français ou étrangers, des laboratoires publics ou privés. 


\title{
Mathematical and numerical study of a system of conservation laws
}

\author{
R. Eymard* and E. Tillier ${ }^{\dagger}$
}

October 18, 2006

\begin{abstract}
The system of equations $(f(u))_{t}-(a(u) v+b(u))_{x}=0$ and $u_{t}-(c(u) v+d(u))_{x}=0$, where the unknowns $u$ and $v$ are functions depending on $(x, t) \in \mathbb{R} \times \mathbb{R}_{+}$, arises within the study of some physical model of the flow of miscible fluids in a porous medium. We give a definition for a weak entropy solution $(u, v)$, inspired by the Liu condition for admissible shocks and by Krushkov entropy pairs. We then prove, in the case of a natural generalization of the Riemann problem, the existence of a weak entropy solution only depending on $x / t$. This property results from the proof of the existence, by passing to the limit on some approximations, of a function $g$ such that $u$ is the classical entropy solution of $u_{t}-((c g+d)(u))_{x}=0$ and simultaneously $w=f(u)$ is the entropy solution of $w_{t}-\left((a g+b)\left(f^{(-1)}(w)\right)\right)_{x}=0$. We then take $v=g(u)$, and the proof that $(u, v)$ is a weak entropy solution of the coupled problem follows from a linear combination of the weak entropy inequalities satisfied by $u$ and $f(u)$. We then show the existence of an entropy weak solution for a general class of data, thanks to the convergence proof of a coupled finite volume scheme. The principle of this scheme is to compute the Godunov numerical flux with some interface functions ensuring the symmetry of the finite volume scheme with respect to both conservation equations.
\end{abstract}

Keywords: nonlinear hyperbolic equations, existence of a solution, Riemann problem, admissible shocks, entropy solutions, convergence of a finite volume scheme.

AMS subject classification: 35A35, 35L45, 65M12

\section{Introduction}

The modelization of the injection of carbon dioxide $\left(\mathrm{CO}_{2}\right)$ into natural underground reservoirs, which seems to be a solution to some environmental problems, involves an increasing number of works (see $[2,4,8,11,13,17]$ and references therein for examples of modelization and simulation works). The present paper presents the mathematical analysis of such a model within a simplified framework. We consider that some $\mathrm{CO}_{2}$ is injected at a given depth in a porous medium saturated with water. For simplicity, we focus on the onedimensional problem resulting from the competition between the migration of this gaseous species by gravity, and its dissolution into water. We denote by $x \in \mathbb{R}$ the vertical space variable, increasing with depth. We denote $X \in[0, \bar{X}]$ the molar fraction of $\mathrm{CO}_{2}$ in water (always comprised between 0 and the maximum dissolution concentration $\bar{X} \in[0,1]$, assumed to be constant) and $S \in[0,1]$ the saturation of water (i.e. the volumic fraction of the porous medium filled by water, the volumic fraction $1-S$ being filled by gaseous $\mathrm{CO}_{2}$ ). These quantities $X$ and $S$, functions of the space variable $x$ and the time variable $t$, are assumed to be such that, for a given $(x, t)$ :

- either the gaseous phase is present, which means that $S(x, t)<1$, and then $X(x, t)=\bar{X}$,

- or the gaseous phase is not present, and then $S(x, t)=1$, and all values $X(x, t) \in[0, \bar{X}]$ are possible. This alternative is summarized into the following relation.

*Université de Marne-la-Vallée, France, Robert.Eymard@univ-mlv.fr

†Institut Français du Pétrole et Université de Marne-la-Vallée, France, Elodie.Tillier@ifp.fr, 


$$
(X(x, t) \leq \bar{X} \text { and } S(x, t)=1) \text { or }(X(x, t)=\bar{X} \text { and } S(x, t) \leq 1) .
$$

We then assume that the transport of $\mathrm{CO}_{2}$ results only from two mechanisms: the flow of the gaseous phase, and the flow of water phase containing some dissolved gas. In this simplified model, we do not take into account the diffusion-dispersion phenomena of $\mathrm{CO}_{2}$ within the water phase, nor the effects of compressibility of the gaseous phase and we assume that the capillary effects are negligible. Under such hypotheses, the conservation equations of the water component and of $\mathrm{CO}_{2}$, given in a dimensionless form, read

$$
\left\{\begin{array}{lll}
{[S(1-X)]_{t}} & -\left[(1-X) k_{w}(S)\left(P_{x}-1\right)\right]_{x} & =0 \\
{[S X+\xi(1-S)]_{t}} & -\left[X k_{w}(S)\left(P_{x}-1\right)+\frac{\xi}{\mu} k_{g}(S)\left(P_{x}-\rho\right)\right]_{x} & =0
\end{array}\right.
$$

where

- the lower index $t$ (resp. $x$ ) denotes the partial derivative with respect to $t$ (resp. $x$ ),

- $\xi$ is the ratio between the molar density of the gas phase and that of water, $\rho$ is the ratio between the bulk density of both phases, $\mu$ is the ratio of viscosity of the gaseous phase and that of the water phase (all these quantities are assumed to be given strictly positive constants),

- $k_{w}(S)$ and $k_{g}(S)$ are respectively the relative permeabilities of the water and the gaseous phases, assumed to be Lipschitz continuous functions of the saturation such that $k_{w}$ is non decreasing with $k_{w}(0)=0$ and $k_{w}(1)=1$, and $k_{g}$ is non increasing with $k_{g}(0)=1$ and $k_{g}(1)=0$, and such that $k_{w}(s)+k_{g}(s)$ is always strictly positive for all $s \in[0,1]$,

- $P(x, t)$ is the common pressure of both phases, function of the space and time variables.

In this model, the following hypothesis is assumed:

$$
\bar{X}<\xi .
$$

Its physical meaning is that a unit volume of gaseous phase contains more moles of $\mathrm{CO}_{2}$ than a unit volume of water containing dissolved $\mathrm{CO}_{2}$ at the maximum concentration. We then introduce the new unknown $u=S X+\xi(1-S)$. Thanks to Hypothesis (3) and using (1), we then express $S$ and $X$ as Lipschitz continuous functions $S(u)$ and $X(u)$ of $u \in[0, \xi]$, given by

$$
\left\{\begin{array}{l}
X(u)=u \text { and } S(u)=1, \forall u \in[0, \bar{X}] \\
X(u)=\bar{X} \text { and } S(u)=\frac{\xi-u}{\xi-\bar{X}}, \forall u \in[\bar{X}, \xi] .
\end{array}\right.
$$

We substitute $S$ and $X$ by $S(u)$ and $X(u)$ in system (2), we introduce the unknown $v(x, t)=P_{x}(x, t)$ and we define the functions

$$
\left\{\begin{array}{l}
f(u)=S(u)(1-X(u)) \\
a(u)=(1-X(u)) k_{w}(S(u)) \\
b(u)=-(1-X(u)) k_{w}(S(u)) \\
c(u)=X(u) k_{w}(S(u))+\frac{\xi}{\mu} k_{g}(S(u)) \\
d(u)=-X(u) k_{w}(S(u))-\frac{\xi}{\mu} k_{g}(S(u)) \rho .
\end{array}\right.
$$

This provides the following system of equations:

$$
\left\{\begin{array}{cl}
(f(u))_{t}-(a(u) v+b(u))_{x}=0 \\
u_{t}-(c(u) v+d(u))_{x}=0 .
\end{array}\right.
$$

Let us first remark that system (6) is not an usual hyperbolic system of equations, since it contains no term $v_{t}$. The first idea to solve this system is to eliminate $u_{t}$ between the two equations. Indeed, this 
could be achieved on a strong formulation of system (6), assuming that $u$ is sufficiently regular and writing $(f(u))_{t}=f^{\prime}(u) u_{t}$. It would then be easy to get $v$ by solving a first order ordinary differential equation, and then one could find a function $\psi$ such that $v(x, t)=\psi(u(x, t), t)$. But reporting such an expression in any of the two equations yields a nonlinear scalar hyperbolic equation with the unknown function $u$, the weak entropy solution of which is in general not continuous (recall that if the weak entropy solution is discontinuous, there does not exist any continuous weak solution). Hence the above method cannot be used for solving this problem in the general case.

Nevertheless, the elimination of $u_{t}$ between the two equations can be done in the particular case where $f(u)$ is an affine function. This is the case if we assume that $\bar{X}=0$ (then $f(u)=1-u / \xi$ ), which then leads to the classical incompressible immiscible two phase flow problem [1]. In this case, it is possible to eliminate the time derivative of the test functions between the weak formulations of both equations. We can then express $v$ as a function of $u$ after a simple integration with respect to $x$ and then get the classical Buckley-Leverett equation. This nonlinear hyperbolic equation has been studied by many authors (see e.g. $[1,3,6]$ and references therein) from the theoretical and numerical points of view. Let us notice that, in this case, discontinuous solutions $u$ and $v$ can be obtained even in the case of initial regular data, which shows the necessity to formulate the problem under a weak formulation.

But $f$ is no longer affine taking $\bar{X}>0$, since $f$ is then a continuous piecewise affine function with a significant slope variation. It then becomes impossible to proceed to an elimination of the time derivative of the test functions on weak formulations of system (6), which prevents from expressing, at each time $t, v$ as a function of $u$. In fact, one cannot expect that such an expression exists in the general case. Indeed, we show, on an analytical example given in section 2 (inspired by the problem presented in the beginning of this introduction), that we can observe the existence, for some times $t>0$, of points $x_{1}$ and $x_{2}$ such that $v\left(x_{1}, t\right) \neq v\left(x_{2}, t\right)$ although $u\left(x_{1}, t\right)=u\left(x_{2}, t\right)$ holds.

System (6) must therefore be solved in a coupled way, including weak formulation senses in order to take into account discontinuous solutions. We obtain a first simple weak sense by multiplying the two equations of (6) by a regular test function, and integrating by parts. The particular case, obtained when $f$ is an affine function, shows that this weak sense cannot be expected to characterize the solution. We have therefore used in section 2 some works of Liu (see [16, 15, 14]) for deriving a notion of entropy weak solution for this system. Then, considering a generalized Riemann problem (the situation is not symmetric with respect to $u$ and $v$ ), we prove that system (6) can be solved thanks to the solution of two nonlinear scalar hyperbolic equations in $u$, the nonlinear functions in each of these equations being linked in order to provide the same shocks and characteristic velocities (theorem 2.5). Note that such a generalized Riemann problem has been studied in a case of multiphase flow in a porous medium, leading to the determination of the shocks and the rarefaction waves in some physical situations [5]. Then, the Liu condition of admissibility of the shocks happens to result from a simple linear combination of the entropy inequalities for both nonlinear hyperbolic equations, using Krushkov entropy pairs [12]. We then build analytically such two functions on some examples in section 2 . We can then provide a proof of the existence, in the general case, of these two nonlinear functions (the uniqueness of which remains at this time an open problem), which relies on the construction of simultaneous convex and concave hulls for two functions (section 3) by passing to the limit on approximate piecewise affine hulls (theorem 2.6).

In order to extend this existence result to more general data than generalized Riemann problems, we then give a finite volume numerical scheme, the convergence of which to an entropy weak solution is proven in section 4. Since we have been able in section 2 to state the existence of the solution of a generalized Riemann problem, it would have been indeed natural to look for a numerical scheme obtained by averaging (after a discrete time step) the solution obtained from the analytical solution, deduced from the resolution of a sequence of generalized Riemann problems. Unfortunately, such an approach does not simultaneously respect both conservation equations, and its convergence properties do not seem to be clear. Thus we have developed an original numerical scheme, defined in such a way that values are defined for $u$ at all the interfaces of the mesh using the Godunov numerical flux, simultaneously respecting the discrete balances resulting from a finite volume scheme applied to both equations. This choice enables the proof 
of a bounded variation estimate, and the proof of the convergence of the scheme to a weak solution of both equation can then be completed. The convergence property is then obtained for a strong topology for $u$, but only for a weak one for $v$. It is worth noticing that the proof of the $L^{\infty}$ estimates on $v$, and that on $u$, cannot hold without the proof of the bounded variation estimate. Thanks to this convergence result, we are therefore able to prove the existence of a solution to system (6) for a large class of initial data (theorem 2.7).

So it is possible to compare the numerical results given by the scheme of section 4 and the analytical solution given in section 2. This is the aim of section 5, where an excellent agreement between these results seems to be a good indication for generalizing the numerical scheme studied here to less simplified models.

\section{Entropy solutions and generalized Riemann problem}

In order to give an entropy weak sense for a solution to system (6), we first state the following hypotheses on the functions $a, b, c, d$ and $f$, always satisfied if these functions are given by (5) defining suitable prolongments.

$$
\left\{\begin{array}{l}
f, a, b, c, d \text { are Lipschitz continuous functions defined on } \mathbb{R}, \\
C_{\text {Lip }}=\max \left(\left\|a^{\prime}\right\|_{\infty},\left\|b^{\prime}\right\|_{\infty},\left\|c^{\prime}\right\|_{\infty},\left\|d^{\prime}\right\|_{\infty},\left\|f^{\prime}\right\|_{\infty}\right) \\
a, b, c, d \text { are bounded on } \mathbb{R} \text { and } C_{\max }=\max \left(\|a\|_{\infty},\|b\|_{\infty},\|c\|_{\infty},\|d\|_{\infty}\right) \\
\text { there exists } f_{m}>0 \text { such that, for a.e. } s \in \mathbb{R},-f^{\prime}(s) \geq f_{m} \\
a(s) \geq 0 \text { and } c(s) \geq 0 \text { for all } s \in \mathbb{R}, \\
\text { there exists } m_{0}>0 \text { such that, for all } s \in \mathbb{R}, a(s)+c(s) f_{m} \geq m_{0}
\end{array}\right.
$$

Remark 2.1 In the framework of the physical problem given in introduction to this paper, the monotony property of $f$ is related to the fact that an increase of $\mathrm{CO}_{2}$ must imply a decrease of water content. The hypothesis $a(s)+c(s) f_{m} \geq m_{0}>0$ expresses the fact that, whatever the water and $\mathrm{CO}_{2}$ contents, the mixture of fluids must remain mobile.

Let us now define some hypotheses on the initial and boundary conditions.

$$
\left\{\begin{array}{l}
u_{0} \in L^{\infty}(\mathbb{R}) \cap B V(\mathbb{R}) \text { and } W_{0}=\left\|u_{0}\right\|_{B V(\mathbb{R})}, \\
\text { there exist } \bar{u}_{0}, \bar{M}_{0} \in \mathbb{R} \text { s.t. } u_{0}(x)=\bar{u}_{0} \text { for a.e. } x \in\left(-\infty, \bar{M}_{0}\right), \\
\left(\text { we then denote } U_{m}, U_{M} \in \mathbb{R} \text { s.t. } U_{m} \leq u_{0}(x) \leq U_{M} \text { for a.e. } x \in \mathbb{R}\right), \\
\bar{v}_{0} \in L^{\infty}\left(\mathbb{R}_{+}\right) \text {is given, and we denote } \bar{V}_{0}=\left\|\bar{v}_{0}\right\|_{L^{\infty}\left(\mathbb{R}_{+}\right)} .
\end{array}\right.
$$

In (8), we classically define the set $B V(\mathbb{R})$ by $B V(\mathbb{R})=\left\{u \in L_{\text {loc }}^{1}(\mathbb{R}),\|u\|_{B V(\mathbb{R})}<\infty\right\}$ with $\|u\|_{B V(\mathbb{R})}=$ $\sup \left\{\int_{\mathbb{R}} u(x) \varphi^{\prime}(x) \mathrm{d} x, \varphi \in C_{c}^{1}(\mathbb{R},[-1,1])\right\}$, where for all $E \subset \mathbb{R}^{d}$ with $d \in \mathbb{N}^{\star}$, for all $p \in \mathbb{N} \cup\{\infty\}$ and all $F \subset \mathbb{R}$, we denote by $C_{c}^{p}(E, F)$ the set of the restrictions to $E$ of all $C^{p}$ functions from $\mathbb{R}^{d}$ to $F$ with a compact support.

Remark 2.2 The hypothesis $u_{0} \in B V(\mathbb{R})$ is strongly used in this paper. The other hypotheses are done in order to handle simple boundary conditions. The generalization of the results of this paper to more general boundary conditions, using in particular the results of [19] and [7], will be the object of further works. We assume below that $v(x, t)$ is equal to the boundary condition $\bar{v}_{0}(t)$ for small values $x$. We could as well give this boundary condition for large $x$, with minor changes in this paper.

Note that $U_{m}=\bar{u}_{0}-W_{0}$ and $U_{M}=\bar{u}_{0}+W_{0}$ always satisfy the third item of (8) from the two previous assumptions.

We now give the following definition. 
Definition 2.3 (Weak entropy solution) Under Hypotheses (7) and (8), the pair (u,v) is said to be an entropy weak solution of the problem:

$$
\left\{\begin{array}{l}
(f(u))_{t}-(a(u) v+b(u))_{x}=0 \\
u_{t}-(c(u) v+d(u))_{x}=0 \\
u(., 0)=u_{0} \\
v(x, .)=\bar{v}_{0} \text { for all } x \text { small enough }
\end{array}\right.
$$

if it is such that

- the functions $u$ and $v$ satisfy $u, v \in L^{\infty}\left(\mathbb{R} \times \mathbb{R}_{+}\right)$,

- the first three equations of (9) are satisfied in the following weak sense:

$$
\begin{aligned}
& \int_{\mathbb{R}_{+}} \int_{\mathbb{R}}\left(f(u(x, t)) \varphi_{t}(x, t)-(a(u(x, t)) v(x, t)+b(u(x, t))) \varphi_{x}(x, t)\right) \mathrm{d} x \mathrm{~d} t+\int_{\mathbb{R}} f\left(u_{0}(x)\right) \varphi(x, 0) \mathrm{d} x=0, \\
& \int_{\mathbb{R}_{+}} \int_{\mathbb{R}}\left(u(x, t) \varphi_{t}(x, t)-(c(u(x, t)) v(x, t)+d(u(x, t))) \varphi_{x}(x, t)\right) \mathrm{d} x \mathrm{~d} t+\int_{\mathbb{R}} u_{0}(x) \varphi(x, 0) \mathrm{d} x=0, \\
& \forall \varphi \in C_{c}^{\infty}\left(\mathbb{R} \times \mathbb{R}_{+}, \mathbb{R}\right),
\end{aligned}
$$

- the following entropy inequalities hold:

$$
\begin{aligned}
& a(\kappa)\left(\begin{array}{l}
\int_{\mathbb{R}_{+}} \int_{\mathbb{R}}\left(\begin{array}{l}
(u(x, t) \top \kappa-\kappa) \varphi_{t}(x, t)- \\
\left(\begin{array}{l}
(c(u(x, t) \top \kappa)-c(\kappa)) v(x, t)+ \\
(d(u(x, t) \top \kappa)-d(\kappa))
\end{array}\right) \varphi_{x}(x, t)
\end{array}\right) \mathrm{d} x \mathrm{~d} t \\
+\int_{\mathbb{R}}\left(u_{0}(x) \top \kappa-\kappa\right) \varphi(x, 0) \mathrm{d} x
\end{array}\right) \\
& -c(\kappa)\left(\begin{array}{l}
\int_{\mathbb{R}_{+}} \int_{\mathbb{R}}\left(\begin{array}{l}
\left(\begin{array}{l}
f(u(x, t) \top \kappa)-f(\kappa)) \varphi_{t}(x, t)- \\
(a(u(x, t) \top \kappa)-a(\kappa)) v(x, t)+ \\
(b(u(x, t) \top \kappa)-b(\kappa))
\end{array}\right) \varphi_{x}(x, t)
\end{array}\right) \mathrm{d} x \mathrm{~d} t \\
+\int_{\mathbb{R}}\left(f\left(u_{0}(x) \top \kappa\right)-f(\kappa)\right) \varphi(x, 0) \mathrm{d} x
\end{array}\right) \geq 0,
\end{aligned}
$$

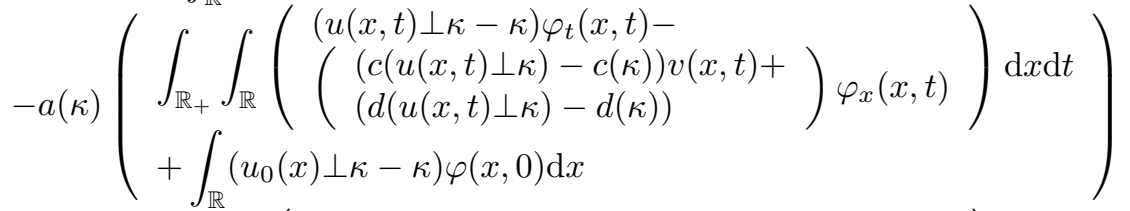

$$
\begin{aligned}
& \left.+c(\kappa)\left(\begin{array}{l}
\int_{\mathbb{R}_{+}} \int_{\mathbb{R}}\left(\begin{array}{l}
\left(\begin{array}{l}
f(u(x, t) \perp \kappa)-f(\kappa)) \varphi_{t}(x, t)- \\
(a(u(x, t) \perp \kappa)-a(\kappa)) v(x, t)+ \\
(b(u(x, t) \perp \kappa)-b(\kappa))
\end{array}\right. \\
+\int_{\mathbb{R}^{\prime}}\left(f\left(u_{0}(x) \perp \kappa\right)-f(\kappa)\right) \varphi(x, 0) \mathrm{d} x
\end{array}\right) \varphi_{x}(x, t)
\end{array}\right) \mathrm{d} x \mathrm{~d} t\right) \geq 0, \\
& \forall \kappa \in \mathbb{R}, \forall \varphi \in C_{c}^{\infty}\left(\mathbb{R} \times \mathbb{R}_{+}, \mathbb{R}_{+}\right),
\end{aligned}
$$

where we denote by $x \top y=\max (x, y)$ and $x \perp y=\min (x, y)$, for all $x, y \in \mathbb{R}$,

- the fourth equation of (9) is satisfied in the following sense:

$$
\forall T>0, \exists M \in \mathbb{R}, v(x, t)=\bar{v}_{0}(t) \text { for a.e. }(x, t) \in(-\infty, M) \times(0, T) .
$$

Let us comment the entropy weak sense (11) taken in the above definition. Let us assume that, at some given time, the solution is such that $u \rightarrow u_{l}$ and $v \rightarrow v_{l}$ for $x \rightarrow x_{0}$ with $x<x_{0}$ and that $u \rightarrow u_{r}$ and $v \rightarrow v_{r}$ for $x \rightarrow x_{0}$ with $x>x_{0}$. Then, the Rankine-Hugoniot relations deduced from system (9) gives the existence of some velocity $V$ such that

$$
\begin{array}{ll}
V\left(f\left(u_{l}\right)-f\left(u_{r}\right)\right) & =-\left(a\left(u_{l}\right) v_{l}+b\left(u_{l}\right)-a\left(u_{r}\right) v_{r}-b\left(u_{r}\right)\right) \\
V\left(u_{l}-u_{r}\right) & =-\left(c\left(u_{l}\right) v_{l}+d\left(u_{l}\right)-c\left(u_{r}\right) v_{r}-d\left(u_{r}\right)\right) .
\end{array}
$$


The Liu criterion, defining an admissible shock [16], expresses that the shock $u_{l} \rightarrow u_{r}$ cannot split in two shocks $u_{l} \rightarrow \kappa, \kappa \rightarrow u_{r}$, for any $\kappa \in \overline{\mathrm{I}}\left(u_{l}, u_{r}\right)$, where we define

$$
\forall s_{1}, s_{2} \in \mathbb{R}, \overline{\mathrm{I}}\left(s_{1}, s_{2}\right)=\left[s_{1}, s_{2}\right] \text { if } s_{1} \leq s_{2} \text {, else } \overline{\mathrm{I}}\left(s_{1}, s_{2}\right)=\left[s_{2}, s_{1}\right] \text {. }
$$

This means that, if $V_{l}, V_{r}$ and $v_{\kappa}$ are reals such that

$$
\begin{array}{ll}
V_{l}\left(f\left(u_{l}\right)-f(\kappa)\right) & =-\left(a\left(u_{l}\right) v_{l}+b\left(u_{l}\right)-a(\kappa) v_{\kappa}-b(\kappa)\right) \\
V_{l}\left(u_{l}-\kappa\right) & =-\left(c\left(u_{l}\right) v_{l}+d\left(u_{l}\right)-c(\kappa) v_{\kappa}-d(\kappa)\right), \\
V_{r}\left(f(\kappa)-f\left(u_{r}\right)\right) & =-\left(a(\kappa) v_{\kappa}+b(\kappa)-a\left(u_{r}\right) v_{r}-b\left(u_{r}\right)\right) \\
V_{r}\left(\kappa-u_{r}\right) & =-\left(c(\kappa) v_{\kappa}+d(\kappa)-c\left(u_{r}\right) v_{r}-d\left(u_{r}\right)\right),
\end{array}
$$

then the properties $V_{l} \geq V$ and $V_{r} \leq V$ must hold. It is then easy to eliminate $v_{\kappa}$ by multiplying the first and the third above equations by $c(\kappa)$, the second and the fourth by $a(\kappa)$, and then subtract the second to the first and the fourth to the third. The inequalities $V_{l} \geq V$ and $V_{r} \leq V$ can then be seen as Rankine-Hugoniot inequalities provided by weak formulation inequalities, in the same way as similar inequalities hold from the entropy weak formulation of a nonlinear scalar hyperbolic inequality using the entropy pairs of Krushkov (see [20, 18, 19, 7]). Hence (11) can be deduced by analogy.

Remark 2.4 In the case where there exist $\alpha, \beta \in \mathbb{R}$ with $f(u)=\alpha u+\beta$, we can easily deduce from definition (2.3) that $v(x, t)$ is obtained from $u(x, t)$ by

$$
v(x, t)=\frac{\left(a\left(\bar{u}_{0}\right)-\alpha c\left(\bar{u}_{0}\right)\right) \bar{v}_{0}(t)+b\left(\bar{u}_{0}\right)-b(u(x, t))-\alpha\left(d\left(\bar{u}_{0}\right)-d(u(x, t))\right)}{a(u(x, t))-\alpha c(u(x, t))}, \text { for a.e. }(x, t) \in \mathbb{R} \times \mathbb{R}_{+},
$$

and $u$ is the unique entropy solution of the equation

$$
u_{t}-\left(c(u) \frac{\left.\left(a\left(\bar{u}_{0}\right)-\alpha c\left(\bar{u}_{0}\right)\right) \bar{v}_{0}(t)+b\left(\bar{u}_{0}\right)-b(u)-\alpha\left(d\left(\bar{u}_{0}\right)-d(u)\right)\right)}{a(u)-\alpha c(u)}+d(u)\right)_{x}=0,
$$

with the initial condition $u(\cdot, 0)=u_{0}$ (it suffices to divide (11) by $\left.a(\kappa)-\alpha c(\kappa)\right)$.

Our aim is now to show that, under particular initial data called "generalized Riemann problem", we can exhibit a weak solution $(u, v)$ to system (9) in the sense of Definition 2.3, only depending on $x / t$, permitting, in some case, to give the analytical expression of this solution. This generalized Riemann problem is defined by three reals $u_{l}, u_{r}$ and $g_{l}$, and by setting $u_{0}(x)=u_{l}$ for a.e. $x<0, u_{0}(x)=u_{r}$ for a.e. $x>0$, and assuming that $v(x, t)=g_{l}$ for small values of $x$ (we again consider a nonsymmetric condition for $v$ ). Let us recall that the concave (resp. convex) hull of a continuous functions $f: \mathbb{R} \rightarrow \mathbb{R}$ on the interval $\left[s_{1}, s_{2}\right]$, for given reals $s_{1} \leq s_{2}$, is the function defined for all $s \in\left[s_{1}, s_{2}\right]$ by the infimum (resp. supremum) value in $s$ of all functions $w \in C^{2}(\mathbb{R})$ such that $w^{\prime \prime} \leq 0$ and $w \geq f$ (resp. $w^{\prime \prime} \geq 0$ and $w \leq f)$ on $\left[s_{1}, s_{2}\right]$. We also recall that, if $f$ is Lipschitz continuous on $\left[s_{1}, s_{2}\right]$, these functions are Lipschitz continuous as well on $\left[s_{1}, s_{2}\right]$, with the same Lipschitz constant. We then state the following sufficient condition for an entropy weak solution to the generalized Riemann problem.

Theorem 2.5 (Generalized Riemann problem) Under Hypotheses (7), using notation (13), let three reals $g_{l}, u_{l}, u_{r}$ be given. Let $g: \overline{\mathrm{I}}\left(u_{l}, u_{r}\right) \rightarrow \mathbb{R}$ be a Lipschitz continuous function such that $g\left(u_{l}\right)=g_{l}$ and such that the functions $\mu, \nu$, defined by $\mu(u)=-(c(u) g(u)+d(u))$ and $\nu(f(u))=-(a(u) g(u)+b(u))$ for all $u \in \overline{\mathrm{I}}\left(u_{l}, u_{r}\right)$, verify $\widehat{\nu}^{\prime}(f(u))=\widehat{\mu}^{\prime}(u)$ for a.e. $u \in \overline{\mathrm{I}}\left(u_{l}, u_{r}\right)$, denoting by $\widehat{\mu}$ is the concave (resp. convex) hull of $\mu$ on $\overline{\mathrm{I}}\left(u_{l}, u_{r}\right)$ and by $\widehat{\nu}$ the convex (resp. concave) hull of $\nu$ on $\overline{\mathrm{I}}\left(f\left(u_{l}\right), f\left(u_{r}\right)\right)$ if $u_{l} \geq u_{r}$ (resp. $\left.u_{l}<u_{r}\right)$. The existence of such a function is stated by theorem 2.6.

Let us define $V_{M}=\operatorname{ess} \sup \widehat{\mu}^{\prime}(s)$ and $V_{m}=\underset{s \in \overline{\mathrm{I}}\left(u_{l}, u_{r}\right)}{\operatorname{ess}} \operatorname{\mu r}^{\prime}(s)$, let $u \in L^{\infty}\left(\mathbb{R} \times \mathbb{R}_{+}\right)$be defined by

$$
\begin{aligned}
& s \in \overline{\mathrm{I}}\left(u_{l}, u_{r}\right) \\
& u(x, t)=u_{l}, \forall t \in(0,+\infty), \text { for a.e. } x \in\left(-\infty, t V_{m}\right) \\
& x=t \widehat{\mu}^{\prime}(u(x, t)), \forall t \in(0,+\infty), \text { for a.e. } x \in\left(t V_{m}, t V_{M}\right) \\
& u(x, t)=u_{r}, \forall t \in(0,+\infty), \text { for a.e. } x \in\left(t V_{M},+\infty\right),
\end{aligned}
$$


and let $v \in L^{\infty}\left(\mathbb{R} \times \mathbb{R}_{+}\right)$be defined by $v(x, t)=g(u(x, t))$ for all $t \in(0,+\infty)$ and a.e. $x \in \mathbb{R}$.

Then $(u, v)$ is an entropy weak solution of the system (9) in the sense of definition 2.3, where hypotheses (8) are satisfied setting $u_{0}(x)=u_{l}$ for a.e. $x<0$ and $u_{0}(x)=u_{r}$ for a.e. $x>0, W_{0}=\left|u_{l}-u_{r}\right|, \bar{u}_{0}=u_{l}$, $\bar{M}_{0}=0$ and $\bar{v}_{0}(t)=g_{l}$ for a.e. $t \in \mathbb{R}_{+}$.

Proof. Thanks to (14) and to the definition of $\widehat{\mu}$, we get from e.g. [20] that $u$ is the unique entropy weak solution of the problem

$$
\begin{aligned}
& u_{t}+(\mu(u))_{x}=u_{t}-(c(u) g(u)+d(u))_{x}=0 \\
& u(., 0)=u_{0} .
\end{aligned}
$$

Therefore it satisfies the classical weak sense, which is a consequence of (17),

$$
\begin{aligned}
& \int_{\mathbb{R}_{+}} \int_{\mathbb{R}}\left(u(x, t) \varphi_{t}(x, t)+\mu(u(x, t)) \varphi_{x}(x, t)\right) \mathrm{d} x \mathrm{~d} t+\int_{\mathbb{R}} u_{0}(x) \varphi(x, 0) \mathrm{d} x=0, \\
& \forall \varphi \in C_{c}^{\infty}\left(\mathbb{R} \times \mathbb{R}_{+}, \mathbb{R}\right),
\end{aligned}
$$

and it also satisfies

$$
\begin{aligned}
& \int_{\mathbb{R}_{+}} \int_{\mathbb{R}}\left((u(x, t) \top \kappa-\kappa) \varphi_{t}(x, t)+(\mu(u(x, t) \top \kappa)-\mu(\kappa)) \varphi_{x}(x, t)\right) \mathrm{d} x \mathrm{~d} t \\
& +\int_{\mathbb{R}}\left(u_{0}(x) \top \kappa-\kappa\right) \varphi(x, 0) \mathrm{d} x \geq 0, \\
& \int_{\mathbb{R}_{+}} \int_{\mathbb{R}}\left((\kappa-u(x, t) \perp \kappa) \varphi_{t}(x, t)+(\mu(\kappa)-\mu(u(x, t) \perp \kappa)) \varphi_{x}(x, t)\right) \mathrm{d} x \mathrm{~d} t \\
& +\int_{\mathbb{R}}\left(\kappa-u_{0}(x) \perp \kappa\right) \varphi(x, 0) \mathrm{d} x \geq 0, \\
& \forall \kappa \in \mathbb{R}, \forall \varphi \in C_{c}^{\infty}\left(\mathbb{R} \times \mathbb{R}_{+}, \mathbb{R}_{+}\right) .
\end{aligned}
$$

Similarly, we get that $f(u)$ is the unique entropy weak solution of the problem

$$
\begin{aligned}
& (f(u))_{t}+(\nu(f(u)))_{x}=(f(u))_{t}-(a(u) g(u)+b(u))_{x}=0 \\
& u(., 0)=u_{0},
\end{aligned}
$$

which implies

$$
\begin{aligned}
& \int_{\mathbb{R}_{+}} \int_{\mathbb{R}}\left(f(u(x, t)) \varphi_{t}(x, t)+\nu(f(u(x, t))) \varphi_{x}(x, t)\right) \mathrm{d} x \mathrm{~d} t+\int_{\mathbb{R}} f\left(u_{0}(x)\right) \varphi(x, 0) \mathrm{d} x=0, \\
& \forall \varphi \in C_{c}^{\infty}\left(\mathbb{R} \times \mathbb{R}_{+}, \mathbb{R}\right),
\end{aligned}
$$

and

$$
\begin{aligned}
& \int_{\mathbb{R}_{+}} \int_{\mathbb{R}}\left((f(u(x, t)) \top f(\kappa)-f(\kappa)) \varphi_{t}(x, t)+(\nu(f(u(x, t)) \top f(\kappa))-\nu(f(\kappa))) \varphi_{x}(x, t)\right) \mathrm{d} x \mathrm{~d} t \\
& +\int_{\mathbb{R}}\left(f\left(u_{0}(x)\right) \top f(\kappa)-f(\kappa)\right) \varphi(x, 0) \mathrm{d} x \geq 0, \\
& \int_{\mathbb{R}_{+}} \int_{\mathbb{R}}\left((f(\kappa)-f(u(x, t)) \perp f(\kappa)) \varphi_{t}(x, t)+(\nu(f(\kappa))-\nu(f(u(x, t)) \perp f(\kappa))) \varphi_{x}(x, t)\right) \mathrm{d} x \mathrm{~d} t \\
& +\int_{\mathbb{R}}\left(f(\kappa)-f\left(u_{0}(x)\right) \perp f(\kappa)\right) \varphi(x, 0) \mathrm{d} x \geq 0, \\
& \forall \kappa \in \mathbb{R}, \forall \varphi \in C_{c}^{\infty}\left(\mathbb{R} \times \mathbb{R}_{+}, \mathbb{R}_{+}\right) .
\end{aligned}
$$

We first note that replacing $\mu(u(x, t))$ by $-c(u(x, t)) v(x, t)-d(u(x, t))$ in $(16)$ and $\nu(f(u(x, t)))$ by $-a(u(x, t)) v(x, t)-b(u(x, t))$ in (19) gives (10). We then remark that $f\left(s_{1} \top s_{2}\right)=$ $f\left(s_{1}\right) \perp f\left(s_{2}\right), f\left(s_{1} \perp s_{2}\right)=f\left(s_{1}\right) \top f\left(s_{2}\right)$ for all reals $s_{1}, s_{2}$. Defining $\operatorname{sign}^{+}(x)=1$ for all $x>0$ else $\operatorname{sign}^{+}(x)=0$ and $\operatorname{sign}^{-}(x)=-1$ for all $x<0$ else $\operatorname{sign}^{-}(x)=0$, we can write the relations

$$
\begin{aligned}
& \mu(u(x, t) \top \kappa)-\mu(\kappa)=-\operatorname{sign}^{+}(u(x, t)-\kappa)(c(u(x, t)) v(x, t)+d(u(x, t))-c(\kappa) g(\kappa)-d(\kappa)), \\
& \mu(\kappa)-\mu(u(x, t) \perp \kappa)=-\operatorname{sign}^{-}(u(x, t)-\kappa)(c(u(x, t)) v(x, t)+d(u(x, t))-c(\kappa) g(\kappa)-d(\kappa)), \\
& \nu(f(u(x, t)) \top f(\kappa))-\nu(f(\kappa))=\operatorname{sign}^{-}(u(x, t)-\kappa)(a(u(x, t)) v(x, t)+b(u(x, t))-a(\kappa) g(\kappa)-b(\kappa)), \\
& \nu(f(\kappa))-\nu(f(u(x, t)) \perp f(\kappa))=\operatorname{sign}^{+}(u(x, t)-\kappa)(a(u(x, t)) v(x, t)+b(u(x, t))-a(\kappa) g(\kappa)-b(\kappa)) .
\end{aligned}
$$


Using the above relations, we get that the sum of the first relation of (17) multiplied by $a(\kappa)$ and of the second relation of (20) multiplied by $c(\kappa)$ provides the first relation of (11), whereas the sum of the second relation of (17) multiplied by $a(\kappa)$ and of the first relation of (20) multiplied by $c(\kappa)$ provides the second relation of (11). Relation (12) is an immediate consequence of

$$
\forall T>0, u(x, t)=u_{l} \text { for a.e. }(x, t) \in\left(-\infty, \min \left(V_{m} T, 0\right)\right) \times(0, T),
$$

and of $g\left(u_{l}\right)=g_{l}$.

\section{An analytical example}

We now apply theorem 2.5 to some particular case, for which we can give the analytical expression of the solutions that we provide. The interest of this example is twofold. First, it follows as closely as possible realistic data in the case of injection of $\mathrm{CO}_{2}$ in a porous medium saturated with water. Secondly, it shows that $v$ cannot be expressed, in the general case, as a function of $u$. We consider the functions $a, b$, $c, d$, and $f$, defined by (4) and (5), and the following data:

$$
\begin{array}{|llllll|}
\xi=0.07 & \bar{X}=0.06 & \rho=0.17 & \mu=0.1 & k_{w}(S)=S & k_{g}(S)=(1-S) \\
\hline
\end{array}
$$

\section{First example}

We consider the case $u_{l}^{(1)}=0$ and $u_{r}^{(1)}=S_{0} \bar{X}+\xi\left(1-S_{0}\right)$ with $S_{0}=0.1$. Hence the left part (the upper one) is fully saturated with pure water, whereas the right one (the lower one) is initially filled by the water phase, at the water saturation $S_{0}$, containing dissolved gas at the maximum concentration, and by the gaseous phase, at the gas saturation $1-S_{0}$. This case corresponds to a zoom on the top of the region in which some $\mathrm{CO}_{2}$ has been previously injected. We assume that the water phase does not move at the top of the region, which corresponds to set the gradient of the pressure equal to the hydrostatic one $g_{l}^{(1)}=1$. Let us denote by $\tau_{f s}^{(1)}=\frac{1-\bar{X}}{\xi-\bar{X}}=-f^{\prime}(u)$, for all $u \in(\bar{X}, \xi)$. It is then possible to find the set of values $\left(g^{(1)}, u_{s}^{(1)}, g_{s}^{(1)}, V_{s}^{(1)}, Q_{s}^{(1)}\right)$, with $u_{s}^{(1)} \in(\bar{X}, \xi)$, solution of the following system of equations:

$$
\left\{\begin{array}{l}
V_{s}^{(1)}\left(f\left(u_{l}^{(1)}\right)-f\left(u_{s}^{(1)}\right)\right)=-\left(a\left(u_{l}^{(1)}\right) g_{l}^{(1)}+b\left(u_{l}^{(1)}\right)-a\left(u_{s}^{(1)}\right) g_{s}^{(1)}-b\left(u_{s}^{(1)}\right)\right), \\
V_{s}^{(1)}\left(u_{l}^{(1)}-u_{s}^{(1)}\right)=-\left(c\left(u_{l}^{(1)}\right) g_{l}^{(1)}+d\left(u_{l}^{(1)}\right)-c\left(u_{s}^{(1)}\right) g_{s}^{(1)}-d\left(u_{s}^{(1)}\right)\right) \\
Q_{s}^{(1)}=\left(a\left(u_{s}^{(1)}\right)+\tau_{f s}^{(1)} c\left(u_{s}^{(1)}\right)\right) g_{s}^{(1)}+b\left(u_{s}^{(1)}\right)+\tau_{f s}^{(1)} d\left(u_{s}^{(1)}\right) \\
\forall u \in\left[\bar{X}, u_{r}^{(1)}\right],\left(a(u)+\tau_{f s}^{(1)} c(u)\right) g^{(1)}(u)+b(u)+\tau_{f s}^{(1)} d(u)=Q_{s}^{(1)} \\
g^{(1)}(u)=g_{l}^{(1)}, \forall u \in[0, \bar{X}] \\
\mu^{\prime}\left(u_{s}^{(1)}\right)=\left(c g^{(1)}+d\right)^{\prime}\left(u_{s}^{(1)}\right)=V_{s}^{(1)}
\end{array}\right.
$$

The function $\left(\widehat{\mu}^{(1)}\right)^{\prime}$ is then given by

$$
\left\{\begin{array}{l}
\left(\widehat{\mu}^{(1)}\right)^{\prime}(u)=V_{s}^{(1)}, \forall u \in\left[u_{l}, u_{s}^{(1)}\right], \\
\left(\widehat{\mu}^{(1)}\right)^{\prime}(u)=\left(c g^{(1)}+d\right)^{\prime}(u), \forall u \in\left[u_{s}^{(1)}, \xi\right]
\end{array}\right.
$$

Hence the solution is given by a shock between $u_{l}^{(1)}$ and $u_{s}^{(1)}$, which moves at the velocity $V_{s}^{(1)}$, and a rarefaction wave between $u_{s}^{(1)}$ and $u_{r}^{(1)}$. In this particular case, it is even possible to give an explicit value for $u_{s}^{(1)}$ :

$$
u_{s}^{(1)}=\frac{\xi \bar{X}(1-\mu)-(\bar{X}-\xi)(\mu \xi \bar{X})^{1 / 2}}{\xi-\mu \bar{X}}
$$

and all the other values are then easily deduced. The functions $g^{(1)}, \mu^{(1)}$ and $\nu^{(1)}$ are represented on Figure 1. Since, by construction, in the case $u_{l}=u_{l}^{(1)}, u_{r}=u_{r}^{(1)}, g_{l}=g_{l}^{(1)}$, the function $g=g^{(1)}$ satisfies the hypotheses of Theorem 2.5, we thus obtain that the pair of functions $\left(u^{(1)}, v^{(1)}\right)$ given by (14) with 
$g=g^{(1)}$, is an entropy weak solution of the system (9) in the sense of definition 2.3. We then get that the value of $g^{(1)}\left(u_{r}^{(1)}\right)$, denoted by $g_{r}^{(1)}$, is given by

$$
g_{r}^{(1)}=\frac{Q_{s}^{(1)}-b\left(u_{r}^{(1)}\right)-\tau_{f s}^{(1)} d\left(u_{r}^{(1)}\right)}{a\left(u_{r}^{(1)}\right)+\tau_{f s}^{(1)} c\left(u_{r}^{(1)}\right)} .
$$

\section{Second example}

Let now consider a second example, coupled with the preceding one (we give hereafter a situation where both examples are simultaneously encountered). In this second problem, we set $u_{l}^{(2)}=u_{r}^{(1)}=S_{0} \bar{X}+$ $\xi\left(1-S_{0}\right), u_{r}^{(2)}=u_{l}^{(1)}=0$, and we set $g_{l}^{(2)}=g_{r}^{(1)}$. In this case, the left part (the upper one) is initially filled by the water phase with dissolved gas and by the gaseous phase, whereas the right one (the lower one) is initially filled by pure water. This case corresponds to a zoom on the bottom of the region in which some $\mathrm{CO}_{2}$ has been previously injected. We then define $u_{s}^{(2)}$ by $u_{s}^{(2)}=\bar{X}$, this value being such that a shock occurs between $u_{l}^{(2)}$ and $u_{s}^{(2)}$. Then the velocity of the shock $V_{s}^{(2)}$ is given by the solution $\left(V_{s}^{(2)}, g_{s}^{(2)}\right)$ of the system

$$
\left\{\begin{array}{l}
V_{s}^{(2)}\left(f\left(u_{l}^{(2)}\right)-f\left(u_{s}^{(2)}\right)\right)=-\left(a\left(u_{l}^{(2)}\right) g_{l}^{(2)}+b\left(u_{l}^{(2)}\right)-a\left(u_{s}^{(2)}\right) g_{s}^{(2)}-b\left(u_{s}^{(2)}\right)\right) \\
V_{s}^{(2)}\left(u_{l}^{(2)}-u_{s}^{(2)}\right)=-\left(c\left(u_{l}^{(2)}\right) g_{l}^{(2)}+d\left(u_{l}^{(2)}\right)-c\left(u_{s}^{(2)}\right) g_{s}^{(2)}-d\left(u_{s}^{(2)}\right)\right) .
\end{array}\right.
$$

Therefore $\left(V_{s}^{(2)}, g_{s}^{(2)}\right)$ is given by

$$
\left\{\begin{array}{l}
V_{s}^{(2)}=\frac{1}{\mu}\left(\rho-g_{l}^{(2)}\right) \\
g_{s}^{(2)}=g_{l}^{(2)} \frac{S_{0}(\mu-1)+1}{\mu}+\left(1-S_{0}\right)\left(1-\frac{\rho}{\mu}\right)
\end{array}\right.
$$

We then define the function $g^{(2)}(u)$, for all $u \in\left[u_{s}^{(2)}, u_{l}^{(2)}\right]$ by

$$
\left\{\begin{array}{l}
V_{s}^{(2)}\left(f\left(u_{l}^{(2)}\right)-f(u)\right)=-\left(a\left(u_{l}^{(2)}\right) g_{l}^{(2)}+b\left(u_{l}^{(2)}\right)-a(u) g^{(2)}(u)-b(u)\right) \\
V_{s}^{(2)}\left(u_{l}^{(2)}-u\right)=-\left(c\left(u_{l}^{(2)}\right) g_{l}^{(2)}+d\left(u_{l}^{(2)}\right)-c(u) g^{(2)}(u)-d(u)\right) .
\end{array}\right.
$$

Since, for all $u \in\left[u_{r}^{(2)}, u_{s}^{(2)}\right]$, the conservation equations are respectively linear with respect to $u$ and $f(u)$, there is a contact discontinuity whose velocity $V_{c}^{(2)}$ is given by $V_{c}^{(2)}=\left(1-g_{s}^{(2)}\right)$. We then define $g^{(2)}(u)=g_{s}^{(2)}$ for all $u \in\left[u_{r}^{(2)}, u_{s}^{(2)}\right]$, and we set $g_{r}^{(2)}=g_{s}^{(2)}$. The function $\left(\widehat{\mu}^{(2)}\right)^{\prime}$ is then given by

$$
\left\{\begin{array}{l}
\left(\widehat{\mu}^{(2)}\right)^{\prime}(u)=V_{s}^{(2)}, \forall u \in\left(u_{s}^{(2)}, u_{l}^{(2)}\right) \\
\left(\widehat{\mu}^{(2)}\right)^{\prime}(u)=V_{c}^{(2)}, \forall u \in\left(u_{r}^{(2)}, u_{s}^{(2)}\right)
\end{array}\right.
$$

The functions $g^{(2)}, \mu^{(2)}$ and $\nu^{(2)}$ are represented on Figure 2. Since, in the case $u_{l}=u_{l}^{(2)}, u_{r}=u_{r}^{(2)}$, $g_{l}=g_{l}^{(2)}$, the function $g=g^{(2)}$ again satisfies the hypotheses of Theorem 2.5, we thus obtain that the pair of functions $\left(u^{(2)}, v^{(2)}\right)$ given by (14) with $g=g^{(2)}$, is an entropy weak solution of the system (9) in the sense of definition 2.3 .

\section{A third example built with the two preceding ones}

It is now possible to consider the case of Problem (9), where the function $u_{0}$ of (8) is given by $u_{0}(x)=u_{l}^{(1)}$ for all $x<2 / 5, u_{0}(x)=u_{r}^{(1)}=u_{l}^{(2)}$ for $x \in(2 / 5,4 / 5)$ and $u_{0}(x)=u_{r}^{(2)}=u_{l}^{(1)}$ for $x>4 / 5$. We then assume that $\bar{v}_{0}(t)=g_{l}^{(1)}$, for a.e. $t \in \mathbb{R}_{+}$. These data correspond to the case where some $\mathrm{CO}_{2}$ has been 
previously injected in the region given by $x \in(2 / 5,4 / 5)$ (simulations of this case are also considered in section 5). We then examine the simultaneous displacement of the top of the bubble and its bottom, at least for a limited period of time. We consider the functions $(u, v)$ given by

$$
\begin{aligned}
& u(x, t)=u^{(1)}(x-2 / 5, t) \text { and } v(x, t)=g^{(1)}\left(u^{(1)}(x-2 / 5, t)\right), \forall t \in(0, T), \text { for a.e } x \in(-\infty, 3 / 5), \\
& u(x, t)=u^{(2)}(x-4 / 5, t) \text { and } v(x, t)=g^{(2)}\left(u^{(2)}(x-4 / 5, t)\right), \forall t \in(0, T), \text { for a.e } x \in(3 / 5,+\infty),
\end{aligned}
$$

with $T>0$ small enough such that the two following conditions simultaneously hold

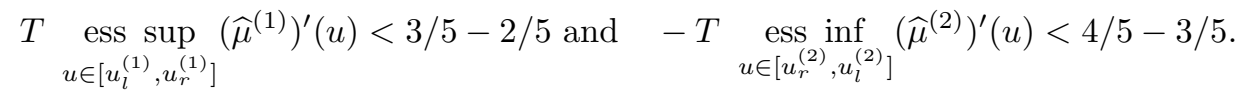

These conditions on $T$ ensure that the solution issued from the first generalized Riemann problem is equal to the initial data for $x>3 / 5$, and that the solution issued from the second one is equal to the initial data for $x<3 / 5$, for all time $t \leq T$. Then this pair $(u, v)$ is an entropy weak solution of the system (9) in the sense of definition 2.3 until time $T$. We see that in this case, the values $v(x, t)$ for small values of $x$ and large ones, correspond to the same value of $u(x, t)$ (which is equal to $\left.u_{l}^{(1)}=u_{r}^{(2)}=0\right)$, are respectively equal to $g_{l}^{(1)}=1$ and $g_{r}^{(2)}=g_{s}^{(2)}$, which are different values in the general case (see the corresponding values on the figures). Note that, although this analytical solution holds only for $t \leq T$, the numerical scheme used in section 5 allows to approximate the solution at any time $t>0$.

We now state the existence result, which allows applying theorem 2.5 to any generalized Riemann problem in the sense given above.

Theorem 2.6 (A coupled convexity property) Under Hypotheses (7), using notation (13), let three reals $g_{l}, u_{l}, u_{r}$ be given.

Then there exists at least one Lipschitz continuous function $g: \overline{\mathrm{I}}\left(u_{l}, u_{r}\right) \rightarrow \mathbb{R}$ such that $g\left(u_{l}\right)=g_{l}$ and such that the functions $\mu, \nu$, defined by $\mu(u)=-(c(u) g(u)+d(u))$ and $\nu(f(u))=-(a(u) g(u)+b(u))$ for all $u \in \overline{\mathrm{I}}\left(u_{l}, u_{r}\right)$, verify $\widehat{\nu}^{\prime}(f(u))=\widehat{\mu}^{\prime}(u)$ for a.e. $u \in \overline{\mathrm{I}}\left(u_{l}, u_{r}\right)$, denoting by $\widehat{\mu}$ the concave (resp. convex) hull of $\mu$ on $\overline{\mathrm{I}}\left(u_{l}, u_{r}\right)$ and by $\widehat{\nu}$ the convex (resp. concave) hull of $\nu$ on $\overline{\mathrm{I}}\left(f\left(u_{l}\right), f\left(u_{r}\right)\right.$ ) if $u_{r} \leq u_{l}$ (resp. $\left.u_{r}>u_{l}\right)$.

The proof of theorem 2.6 is a straightforward consequence of Lemma 3.2 proven in section 3 . Let us conclude this section with the following theorem.

Theorem 2.7 (Existence of an entropy weak solution of the system (9)) Under Hypotheses (7) and (8), there exists at least one entropy weak solution $(u, v)$ of the system (9) in the sense of definition 2.3, which moreover satisfies $u-u_{0} \in \operatorname{Lip}\left(\mathbb{R}_{+} ; L^{1}(\mathbb{R})\right) \cap L^{\infty}\left(\mathbb{R}_{+} ; B V(\mathbb{R})\right)$.

The proof of Theorem 2.7 is given in section 4, by passing to the limit in a finite volume scheme.

\section{Proof of theorem 2.6}

Recall of method for the decoupled case

In order to proceed to the proof of theorem 2.6 by passing to the limit in some approximation method, let us first recall a method to approximate, for two given reals $u_{l}$ and $u_{r}$, the concave $\left(u_{l} \geq u_{r}\right)$ or convex $\left(u_{l} \leq u_{r}\right)$ hull $\widehat{\mu}$ of a function $\mu$ on the interval $\overline{\mathrm{I}}\left(u_{l}, u_{r}\right)$.

For all $i \leq j \in \mathbb{N}$, we denote $\llbracket i, j \rrbracket=\{k \in \mathbb{N}, i \leq k \leq j\}$. Let $N \in \mathbb{N}$ with $N \geq 2$ be given. We define the 
sequences $(\widehat{I}(j))_{j \in \llbracket 0, N \rrbracket}$ and $\left(v_{\widehat{I}(j)}\right)_{j \in \llbracket 0, N \rrbracket, \widehat{I}(j)<N}$ by

$$
\left\{\begin{array}{l}
u_{j}=u_{l}+\frac{j}{N}\left(u_{r}-u_{l}\right), \forall j \in \llbracket 0, N \rrbracket, \\
\widehat{I}(0)=0, \\
\forall j \in \llbracket 0, N-1 \rrbracket, \\
\text { if } \widehat{I}(j)<N \text { then }\left\{\begin{array}{l}
v_{\widehat{I}(j)}=\min _{k \in \llbracket \mathbb{I}(j)+1, N \rrbracket} \frac{\mu\left(u_{k}\right)-\mu\left(u_{\widehat{I}(j)}\right)}{u_{k}-u_{\widehat{I}(j)}}, \\
\widehat{I}(j+1) \text { is any element of }\left\{k \in \llbracket \widehat{I}(j)+1, N \rrbracket, \frac{\mu\left(u_{k}\right)-\mu\left(u_{\widehat{I}(j)}\right)}{u_{k}-u_{\widehat{I}(j)}}=v_{\widehat{I}(j)}\right\} \\
\text { else } \widehat{I}(j+1)=N .
\end{array}\right.
\end{array}\right.
$$

We next complete the definition of the sequence $\left(v_{i}\right)_{i \in \llbracket 0, N-1 \rrbracket}$ by

$$
\left\{\begin{array}{l}
\widehat{N}=\min \{j \in \llbracket 0, N \rrbracket, \widehat{I}(j)=N\} \\
\forall j \in \llbracket 0, \widehat{N}-1 \rrbracket, \forall k \in \llbracket \widehat{I}(j), \widehat{I}(j+1)-1 \rrbracket, v_{k}=v_{\widehat{I}(j)} .
\end{array}\right.
$$

Then the piecewise constant function $\phi^{(N)}$, defined by

$$
\phi^{(N)}: \overline{\mathrm{I}}\left(u_{l}, u_{r}\right) \rightarrow \mathbb{R}, u \mapsto v_{k}, \text { for a.e. } u \in \overline{\mathrm{I}}\left(u_{k}, u_{k+1}\right), \forall k \in \llbracket 0, N-1 \rrbracket,
$$

permits to define the piecewise affine continuous function $\widehat{\mu}^{(N)}$ :

$$
\widehat{\mu}^{(N)}: \overline{\mathrm{I}}\left(u_{l}, u_{r}\right) \rightarrow \mathbb{R}, u \mapsto \mu\left(u_{l}\right)+\int_{u_{l}}^{u} \phi^{(N)}(s) \mathrm{d} s,
$$

which is the concave hull $\left(u_{l} \geq u_{r}\right)$ or the convex hull $\left(u_{l} \leq u_{r}\right)$ of the function $\mu^{(N)}$ which is piecewise affine on all $\overline{\mathrm{I}}\left(u_{k}, u_{k+1}\right), k \in \llbracket 0, N \rrbracket$, such that $\mu^{(N)}\left(u_{k}\right)=\mu\left(u_{k}\right)$ for all $k \in \llbracket 0, N \rrbracket$. Then one can prove that $\mu^{(N)}$ uniformly converges to $\mu$ on $\overline{\mathrm{I}}\left(u_{l}, u_{r}\right)$ as $N \rightarrow \infty$, whereas $\widehat{\mu}^{(N)}$ also uniformly converges to $\widehat{\mu}$ on $\overline{\mathrm{I}}\left(u_{l}, u_{r}\right)$ as $N \rightarrow \infty$ (note that this result is indeed a consequence of Lemma 3.2 below, in the particular case where $c(u)=0$ and $a(u)=1$ for all $u \in \mathbb{R})$. The approximation method used below is then inspired by this one.

\section{Some functions related to the Rankine-Hugoniot relations}

We now define two functions deduced from the Rankine-Hugoniot relations resulting from the conservation laws (6). Let us assume that there exists three reals $u_{1}, u_{2}$ and $g_{1}$ such that $u$ tends to $u_{1}$ and $v$ tends to $g_{1}$ for $x$ tending to $x_{0}$ with $x<x_{0}$ and that $u$ tends to $u_{2}$ for $x$ tending to $x_{0}$ with $x>x_{0}$. Then, from the system of the two Rankine-Hugoniot relations, we can deduce the value $g_{2}$ to which tends $v$ for $x$ tending to $x_{0}$ with $x>x_{0}$, as well as the velocity of the shock. Indeed, this velocity and the value $g_{2}$ are therefore functions of $g_{1}, u_{1}$ and $u_{2}$, respectively denoted $V\left(g_{1}, u_{1}, u_{2}\right)$ and $G\left(g_{1}, u_{1}, u_{2}\right)$, solutions to the following linear system of equations in the case $u_{1} \neq u_{2}$.

$$
\begin{cases}V\left(g_{1}, u_{1}, u_{2}\right)\left(f\left(u_{1}\right)-f\left(u_{2}\right)\right) & =-\left(a\left(u_{1}\right) g_{1}+b\left(u_{1}\right)-a\left(u_{2}\right) G\left(g_{1}, u_{1}, u_{2}\right)-b\left(u_{2}\right)\right) \\ V\left(g_{1}, u_{1}, u_{2}\right)\left(u_{1}-u_{2}\right) & =-\left(c\left(u_{1}\right) g_{1}+d\left(u_{1}\right)-c\left(u_{2}\right) G\left(g_{1}, u_{1}, u_{2}\right)-d\left(u_{2}\right)\right) .\end{cases}
$$

Indeed, thanks to hypotheses (7) and introducing the notation

$$
\tau_{h}\left(u_{1}, u_{2}\right)=\frac{h\left(u_{2}\right)-h\left(u_{1}\right)}{u_{2}-u_{1}}, \forall h \in C^{0}(\mathbb{R}), \forall u_{1}, u_{2} \in \mathbb{R} \text { with } u_{1} \neq u_{2},
$$

(note that we have $\left.-\tau_{f}\left(u_{1}, u_{2}\right) \geq f_{m}\right)$ we get the following expressions for $V\left(g_{1}, u_{1}, u_{2}\right)$ and $G\left(g_{1}, u_{1}, u_{2}\right)$ 
(we prolong the latter by continuity for $u_{1}=u_{2}$ ).

$$
\left\{\begin{array}{l}
V\left(g_{1}, u_{1}, u_{2}\right)=\frac{\left(c\left(u_{2}\right) \tau_{a}\left(u_{1}, u_{2}\right)-a\left(u_{2}\right) \tau_{c}\left(u_{1}, u_{2}\right)\right) g_{1}+c\left(u_{2}\right) \tau_{b}\left(u_{1}, u_{2}\right)-a\left(u_{2}\right) \tau_{d}\left(u_{1}, u_{2}\right)}{a\left(u_{2}\right)-c\left(u_{2}\right) \tau_{f}\left(u_{1}, u_{2}\right)}, \\
G\left(g_{1}, u_{1}, u_{2}\right)=\frac{\left(a\left(u_{1}\right)-c\left(u_{1}\right) \tau_{f}\left(u_{1}, u_{2}\right)\right) g_{1}+b\left(u_{1}\right)-b\left(u_{2}\right)-\left(d\left(u_{1}\right)-d\left(u_{2}\right)\right) \tau_{f}\left(u_{1}, u_{2}\right)}{a\left(u_{2}\right)-c\left(u_{2}\right) \tau_{f}\left(u_{1}, u_{2}\right)}, \\
\forall g_{1}, u_{1}, u_{2} \in \mathbb{R} \text { with } u_{1} \neq u_{2}, \\
G\left(g_{1}, u_{1}, u_{1}\right)=g_{1}, \forall g_{1}, u_{1} \in \mathbb{R} .
\end{array}\right.
$$

Some properties of these functions, used in the next proofs, are given in an appendix.

\section{Approximation in the case of the coupled problem}

Let us now turn to the coupled problem considered in this paper. Let reals $g_{l}, u_{l}, u_{r}$ be given. In order to prove theorem 2.6, we must show the existence of a Lipschitz continuous function $g: \overline{\mathrm{I}}\left(u_{l}, u_{r}\right) \rightarrow \mathbb{R}$ such that $g\left(u_{l}\right)=g_{l}$ and the functions $\mu, \nu$ defined by: $\mu(u)=-(c(u) g(u)+d(u))$ and $\nu(f(u))=$ $-(a(u) g(u)+b(u))$ for all $u \in \overline{\mathrm{I}}\left(u_{l}, u_{r}\right)$ verify $\widehat{\nu}^{\prime}(f(u))=\widehat{\mu}^{\prime}(u)$ for a.e. $u \in \overline{\mathrm{I}}\left(u_{l}, u_{r}\right)$, denoting by $\widehat{\mu}$ is the concave (resp. convex) hull of $\mu$ on $\overline{\mathrm{I}}\left(u_{l}, u_{r}\right)$ and by $\widehat{\nu}$ the convex (resp. concave) hull of $\nu$ on $\overline{\mathrm{I}}\left(f\left(u_{l}\right), f\left(u_{r}\right)\right)$ if $u_{l} \geq u_{r}$ (resp. $u_{l}<u_{r}$ ). We then follow the lines of the approximation method given in introduction to this section. Let $N \in \mathbb{N}$ with $N \geq 2$ be given. Denoting for all $i \leq j \in \mathbb{N}$ by $\llbracket i, j \rrbracket=\{k \in \mathbb{N}, i \leq k \leq j\}$, we first define the sequences $(\widehat{I}(j))_{j \in \llbracket 0, N \rrbracket},\left(v_{\widehat{I}(j)}\right)_{j \in \llbracket 0, N \rrbracket, \widehat{I}(j)<N}$ and $\left(g_{\widehat{I}(j)}\right)_{j \in \llbracket 0, N \rrbracket}$ (these sequences are used to define the approximations of the convex or concave hulls which are looking for) by

$$
\left\{\begin{array}{l}
u_{j}=u_{l}+\frac{j}{N}\left(u_{r}-u_{l}\right), \forall j \in \llbracket 0, N \rrbracket, \\
\widehat{I}(0)=0, g_{0}=g_{l}, \\
\forall j \in \llbracket 0, N-1 \rrbracket, \\
\text { if } \widehat{I}(j)<N \text { then }\left\{\begin{array}{l}
v_{\widehat{I}(j)}=\min _{k \in \llbracket \widehat{I}(j)+1, N \rrbracket} V\left(g_{\widehat{I}(j)}, u_{\widehat{I}(j)}, u_{k}\right), \\
\widehat{I}(j+1) \text { is any element of }\left\{k \in \llbracket \widehat{I}(j)+1, N \rrbracket, V\left(g_{\widehat{I}(j)}, u_{\widehat{I}(j)}, u_{k}\right)=v_{\widehat{I}(j)}\right\}
\end{array},\right. \\
\text { else } \widehat{I}(j+1)=N .
\end{array}\right.
$$

We next complete the definition of the sequences $\left(g_{i}\right)_{i \in \llbracket 0, N \rrbracket}$ and $\left(v_{i}\right)_{i \in \llbracket 0, N-1 \rrbracket}$ by

$$
\left\{\begin{array}{l}
\widehat{N}=\min \{j \in \llbracket 0, N \rrbracket, \widehat{I}(j)=N\} \\
\forall j \in \llbracket 0, \widehat{N}-1 \rrbracket, \forall k \in \llbracket \widehat{I}(j), \widehat{I}(j+1)-1 \rrbracket, g_{k+1}=G\left(g_{\widehat{I}(j)}, u_{\widehat{I}(j)}, u_{k+1}\right) \text { and } v_{k}=v_{\widehat{I}(j)} .
\end{array}\right.
$$

Thanks to the definition of sequences $\left(g_{i}\right)_{i \in \llbracket 0, N \rrbracket}$ and $\left(v_{i}\right)_{i \in \llbracket 0, N-1 \rrbracket}$, we can now define the following piecewise constant functions:

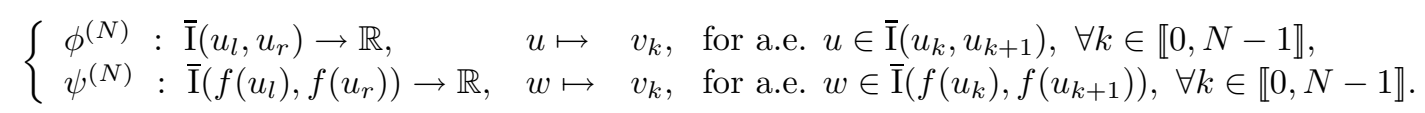

The integration of these piecewise constant functions allows to define the following piecewise affine continuous functions:

$$
\begin{cases}\widehat{\mu}^{(N)}: \overline{\mathrm{I}}\left(u_{l}, u_{r}\right) \rightarrow \mathbb{R}, & u \mapsto-\left(c\left(u_{l}\right) g_{l}+d\left(u_{l}\right)\right)+\int_{u_{l}}^{u} \phi^{(N)}(s) \mathrm{d} s, \\ \widehat{\nu}^{(N)}: \overline{\mathrm{I}}\left(f\left(u_{l}\right), f\left(u_{r}\right)\right) \rightarrow \mathbb{R}, & w \mapsto-\left(a\left(u_{l}\right) g_{l}+b\left(u_{l}\right)\right)+\int_{f\left(u_{l}\right)}^{w} \psi^{(N)}(s) \mathrm{d} s .\end{cases}
$$

We then denote by $g^{(N)}, \mu^{(N)}$ the continuous functions which are piecewise affine on all $\overline{\mathrm{I}}\left(u_{k}, u_{k+1}\right)$, $k \in \llbracket 0, N \rrbracket$, and $\nu^{(N)}$ the continuous function which is piecewise affine on all $\overline{\mathrm{I}}\left(f\left(u_{k}\right), f\left(u_{k+1}\right)\right), k \in \llbracket 0, N \rrbracket$, 
such that

$$
\left\{\begin{array}{lll}
g^{(N)}: \overline{\mathrm{I}}\left(u_{l}, u_{r}\right) \rightarrow \mathbb{R}, & g^{(N)}\left(u_{k}\right)=g_{k}, \forall k \in \llbracket 0, N \rrbracket \\
\mu^{(N)}: \overline{\mathrm{I}}\left(u_{l}, u_{r}\right) \rightarrow \mathbb{R}, & \mu^{(N)}\left(u_{k}\right)=-\left(c\left(u_{k}\right) g_{k}+d\left(u_{k}\right)\right), \forall k \in \llbracket 0, N \rrbracket, \\
\nu^{(N)}: \overline{\mathrm{I}}\left(f\left(u_{l}\right), f\left(u_{r}\right)\right) \rightarrow \mathbb{R}, & \nu^{(N)}\left(f\left(u_{k}\right)\right)=-\left(a\left(u_{k}\right) g_{k}+b\left(u_{k}\right)\right), \quad \forall k \in \llbracket 0, N \rrbracket .
\end{array}\right.
$$

We then have the following property.

Lemma 3.1 Under Hypotheses (7), let three reals $g_{l}, u_{l}, u_{r}$ be given. Let $N \in \mathbb{N}$ with $N \geq 2$ be given and let $\widehat{N} \in \mathbb{N}$ and the sequences $(\widehat{I}(j))_{j \in \llbracket 0, \widehat{N} \rrbracket},\left(u_{j}\right)_{j \in \llbracket 0, N \rrbracket},\left(g_{i}\right)_{i \in \llbracket 0, N \rrbracket}$ and $\left(v_{i}\right)_{i \in \llbracket 0, N-1 \rrbracket}$ be given by (27)-(28). Then the following properties hold:

1. the sequence $\left(v_{\widehat{I}(j)}\right)_{j \in \llbracket 0, \widehat{N}-1 \rrbracket}$, and therefore the sequence $\left(v_{j}\right)_{j \in \llbracket 0, N-1 \rrbracket}$, are non decreasing,

2. the following inequality holds

$$
\begin{aligned}
& \forall j \in \llbracket 0, \widehat{N} \rrbracket, \quad \forall k \in \llbracket \widehat{I}(j)+1, \widehat{I}(j+1)-1 \rrbracket, \\
& V\left(g_{\widehat{I}(j)}, u_{\widehat{I}(j)}, u_{k}\right) \geq V\left(g_{\widehat{I}(j)}, u_{\widehat{I}(j)}, u_{\widehat{I}(j+1)}\right) \geq V\left(g_{k}, u_{k}, u_{\widehat{I}(j+1)}\right),
\end{aligned}
$$

3. if $u_{r}<u_{l}$ (resp. $u_{r}>u_{l}$ ), then the functions $\widehat{\mu}^{(N)}$ is the concave (resp. convex) hull of $\mu^{(N)}$ on $\overline{\mathrm{I}}\left(u_{l}, u_{r}\right)$ and $\widehat{\nu}^{(N)}$ is the the convex (resp. concave) hull of $\nu^{(N)}$ on $\overline{\mathrm{I}}\left(f\left(u_{l}\right), f\left(u_{r}\right)\right)$ (these functions are defined by (30) and (31)).

4. the function $g^{(N)}$ is bounded independently of $N$ and is Lipschitz continuous on $\overline{\mathrm{I}}\left(u_{l}, u_{r}\right)$ with a constant independent of $N$.

5. the sequence $\left(v_{i}\right)_{i \in \llbracket 0, N-1 \rrbracket}$ is bounded independently of $N$.

\section{Proof.}

Proof of item 1

Let us show that the sequence $\left(v_{\widehat{I}(j)}\right)_{j \in \llbracket 0, \widehat{N} \rrbracket}$ is non decreasing. Let $j \in \llbracket 0, \widehat{N}-2 \rrbracket$ and $k \in \llbracket \widehat{I}(j+1)+1, N \rrbracket$ be given. By definition of $v_{\widehat{I}(j)}$, we have $V\left(g_{\widehat{I}(j)}, u_{\widehat{I}(j)}, u_{k}\right) \geq v_{\widehat{I}(j)}=V\left(g_{\widehat{I}(j)}, u_{\widehat{I}(j)}, u_{\widehat{I}(j+1)}\right)$. We apply Lemma 5.2 with $g_{1}=g_{\widehat{I}(j)}, u_{1}=u_{\widehat{I}(j)}, u_{2}=u_{\widehat{I}(j+1)}$ and $u_{3}=u_{k}$. We then get that the sign of $V\left(g_{\widehat{I}(j+1)}, u_{\widehat{I}(j+1)}, u_{k}\right)-V\left(g_{\widehat{I}(j)}, u_{\widehat{I}(j)}, u_{k}\right)$ is the same as that of $V\left(g_{\widehat{I}(j)}, u_{\widehat{I}(j)}, u_{k}\right)-V\left(g_{\widehat{I}(j)}, u_{\widehat{I}(j)}, u_{\widehat{I}(j+1)}\right)$. Thus, the value $v_{\widehat{I}(j)}=V\left(g_{\widehat{I}(j)}, u_{\widehat{I}(j)}, u_{\widehat{I}(j+1)}\right)$, lower than or equal to $V\left(g_{\widehat{I}(j)}, u_{\widehat{I}(j)}, u_{k}\right)$ for $k \in \llbracket \widehat{I}(j+$ 1) $+1, N \rrbracket$, is lower than or equal to $V\left(g_{\widehat{I}(j+1)}, u_{\widehat{I}(j+1)}, u_{k}\right)$. This proves that $v_{\widehat{I}(j+1)} \geq v_{\widehat{I}(j)}$.

Proof of item 2

The left inequality in (32) results from (27). The right one is an immediate consequence of the left one and of Lemma 5.2 with $g_{1}=g_{\widehat{I}(j)}, u_{1}=u_{\widehat{I}(j)}, u_{2}=u_{k}$ and $u_{3}=u_{\widehat{I}(j+1)}$.

Proof of item 3

Let us now assume that $u_{r}<u_{l}$, and let us prove that the function $\widehat{\mu}^{(N)}$ is the concave hull of $\mu^{(N)}$ on $\left[u_{r}, u_{l}\right]$ (the case $u_{r}=u_{l}$ is straightforward, since it leads to constant functions, and the case $u_{r}>u_{l}$ can be handled in a similar way). For all $j \in \llbracket 0, \widehat{N} \rrbracket$, we have $\mu^{(N)}\left(u_{\widehat{I}(j)}\right)=-\left(c\left(u_{\widehat{I}(j)}\right) g_{\widehat{I}(j)}+d\left(u_{\widehat{I}(j)}\right)\right)$. We have

$$
\mu^{(N)}\left(u_{\widehat{I}(j)}\right)-\mu^{(N)}\left(u_{\widehat{I}(j+1)}\right)=c\left(u_{\widehat{I}(j+1)}\right) g_{\widehat{I}(j+1)}-c\left(u_{\widehat{I}(j)}\right) g_{\widehat{I}(j)}+d\left(u_{\widehat{I}(j+1)}\right)-d\left(u_{\widehat{I}(j)}\right)
$$

The algorithm gives $g_{\widehat{I}(j+1)}=G\left(g_{\widehat{I}(j)}, u_{\widehat{I}(j)}, u_{\widehat{I}(j+1)}\right)$, so

$$
\mu^{(N)}\left(u_{\widehat{I}(j)}\right)-\mu^{(N)}\left(u_{\widehat{I}(j+1)}\right)=c\left(u_{\widehat{I}(j+1)}\right) G\left(g_{\widehat{I}(j)}, u_{\widehat{I}(j)}, u_{\widehat{I}(j+1)}\right)-c\left(u_{\widehat{I}(j)}\right) g_{\widehat{I}(j)}+d\left(u_{\widehat{I}(j+1)}\right)-d\left(u_{\widehat{I}(j)}\right),
$$

using the definition of $V$, we obtain

$$
\mu^{(N)}\left(u_{\widehat{I}(j)}\right)-\mu^{(N)}\left(u_{\widehat{I}(j+1)}\right)=V\left(g_{\widehat{I}(j)}, u_{\widehat{I}(j)}, u_{\widehat{I}(j+1)}\right)\left(u_{\widehat{I}(j)}-u_{\widehat{I}(j+1)}\right)
$$


Hence, using the algorithm, we get that, for all $j \in \llbracket 0, \widehat{N}-1 \rrbracket$,

$$
\mu^{(N)}\left(u_{\widehat{I}(j)}\right)-\mu^{(N)}\left(u_{\widehat{I}(j+1)}\right)=v_{\widehat{I}(j)}\left(u_{\widehat{I}(j)}-u_{\widehat{I}(j+1)}\right) .
$$

We have, by construction, $v_{\widehat{I}(j)}=v_{k}$, for all $k \in \llbracket \widehat{I}(j), \widehat{I}(j+1)-1 \rrbracket$ so

$$
\begin{aligned}
\mu^{(N)}\left(u_{\widehat{I}(j)}\right)-\mu^{(N)}\left(u_{\widehat{I}(j+1)}\right) & =v_{\widehat{I}(j)}\left(u_{\widehat{I}(j)}-u_{\widehat{I}(j+1)}\right) \\
& =\int_{u_{\widehat{I}(j+1)}}^{u_{\widehat{I}(j)}} v_{\widehat{I}(j)}=\int_{u_{\widehat{I}(j+1)}} v_{k}=\int_{u_{\widehat{I}(j+1)}}^{u_{\widehat{I}(j)}} \phi^{(N)}(u)
\end{aligned}
$$

This leads to $\mu^{(N)}\left(u_{\widehat{I}(j)}\right)=\widehat{\mu}^{(N)}\left(u_{\widehat{I}(j)}\right)$, thanks to definition of $\mu^{(N)}$. for all $j \in \llbracket 0, \widehat{N} \rrbracket$. Since for all $k \in \llbracket \widehat{I}(j)+1, \widehat{I}(j+1)-1 \rrbracket$, we have $g_{k}=G\left(g_{\widehat{I}(j)}, u_{\widehat{I}(j)}, u_{k}\right)$, we get

$$
\mu^{(N)}\left(u_{\widehat{I}(j)}\right)-\mu^{(N)}\left(u_{k}\right)=V\left(g_{\widehat{I}(j)}, u_{\widehat{I}(j)}, u_{k}\right)\left(u_{\widehat{I}(j)}-u_{k}\right) .
$$

Using (32), we obtain that $\mu^{(N)}\left(u_{\widehat{I}(j)}\right)-\mu^{(N)}\left(u_{k}\right) \geq \widehat{v}_{j}^{(N-1)}\left(u_{\widehat{I}(j)}-u_{k}\right)$. This proves that $\mu^{(N)}\left(u_{k}\right) \leq$ $\widehat{\mu}^{(N)}\left(u_{k}\right)$, and therefore concludes the proof that $\widehat{\mu}^{(N)}$ is the concave hull of $\mu^{(N)}$ on $\left[u_{r}, u_{l}\right]$. Similarly, we get that $\nu^{(N)}\left(f\left(u_{\widehat{I}(j)}\right)\right)=\widehat{\nu}^{(N)}\left(f\left(u_{\widehat{I}(j)}\right)\right)$ for all $j \in \llbracket 0, \widehat{N} \rrbracket$ and that

$$
\nu^{(N)}\left(f\left(u_{\widehat{I}(j)}\right)\right)-\nu^{(N)}\left(f\left(u_{k}\right)\right)=V\left(g_{\widehat{I}(j)}, u_{\widehat{I}(j)}, u_{k}\right)\left(f\left(u_{\widehat{I}(j)}\right)-f\left(u_{k}\right)\right) .
$$

Therefore, since $f$ is strictly decreasing, we get that $\nu^{(N)}\left(f\left(u_{k}\right)\right) \geq \widehat{\nu}^{(N)}\left(f\left(u_{k}\right)\right)$, which shows that $\widehat{\nu}^{(N)}$ is the convex hull of $\nu^{(N)}$ on $\left[f\left(u_{l}\right), f\left(u_{r}\right)\right]$. The case $u_{r}>u_{l}$ yields to similar conclusions.

Proof of item 4

We will first show this item about the sequence $g_{\widehat{I}(j)}, j \in \llbracket 0, \widehat{N} \rrbracket$. Thanks to the definition of $G$, we have $g_{\widehat{I}(j)}=G\left(g_{\widehat{I}(j)}, u_{\widehat{I}(j)}, u_{\widehat{I}(j)}\right)$, and by construction in the algorithm $(27), g_{\widehat{I}(j+1)}=G\left(g_{\widehat{I}(j)}, u_{\widehat{I}(j)}, u_{\widehat{I}(j+1)}\right)$. By applying lemma (5.1), we get

$$
\left|g_{\widehat{I}(j+1)}-g_{\widehat{I}(j)}\right| \leq C_{14}\left(\left|g_{\widehat{I}(j)}\right|+1\right)\left|u_{\widehat{I}(j+1)}-u_{\widehat{I}(j)}\right| .
$$

Using $g_{\widehat{I}(0)}=g_{l}$, and applying the discrete Gronwall's Lemma 5.3, we obtain,

$$
\left|g_{\widehat{I}(j)}\right| \leq\left(\left|g_{l}\right|+1\right) \exp \left[C_{14} \sum_{i=0}^{\widehat{N}-1}\left|u_{\widehat{I}(i+1)}-u_{\widehat{I}(i)}\right|\right],
$$

Since $\sum_{i=0}^{\widehat{N}-1}\left|u_{\widehat{I}(i+1)}-u_{\widehat{I}(i)}\right|=\left|u_{l}-u_{r}\right|$, we get,

$$
\left|g_{\widehat{I}(j)}\right| \leq C_{1},
$$

with $C_{1}=\left(\left|g_{l}\right|+1\right) \exp \left[C_{14}\left|u_{l}-u_{r}\right|\right]-1$. We now turn to the study of the whole sequence $\left(g_{k}\right)_{k \in \llbracket 0, N \rrbracket}$. We remark that, for all $j \in \llbracket 0, \widehat{N} \rrbracket$ and for all $k$ such that $\widehat{I}(j) \leq k \leq \widehat{I}(j+1)-1$, we have

$$
\begin{aligned}
& g_{k}=G\left(g_{\widehat{I}(j)}, u_{\widehat{I}(j)}, u_{k}\right) \\
& g_{k+1}=G\left(g_{\widehat{I}(j)}, u_{\widehat{I}(j)}, u_{k+1}\right) .
\end{aligned}
$$

Hence, for all $k \in \llbracket \widehat{I}(j), \widehat{I}(j+1) \rrbracket$, we get

$$
\left|g_{k+1}-g_{k}\right| \leq C_{14}\left(\left|g_{\widehat{I}(j)}\right|+1\right)\left|u_{k+1}-u_{k}\right|
$$


Since $\left|g_{\widehat{I}(j)}\right| \leq C_{1}$, we conclude that $g_{k}$ is Lipschitz continuous with the constant $C_{2}=C_{14}\left(C_{1}+1\right)$. From inequality (38) and thanks to an easy induction, we then get that

$$
\left|g_{k}\right| \leq\left|g_{l}\right|+C_{14}\left(C_{1}+1\right)\left|u_{r}-u_{l}\right|, \forall k \in \llbracket 0, N \rrbracket,
$$

which provides a bound for $g$, independent of $N$.

Proof of item 5

Thanks to (27)-(28), we get that for all $k \in \llbracket \widehat{I}(j), \widehat{I}(j+1)-1 \rrbracket, v_{k}=\min _{i \in \llbracket \widehat{I}(j)+1, N \rrbracket} V\left(g_{\widehat{I}(j)}, u_{\widehat{I}(j)}, u_{i}\right)$.

Since $\left|g_{k}\right|$ is bounded independently of $N$ for all $k \in \llbracket 0, N \rrbracket$, we get from (26) and (39) that for all $s_{1}, s_{2} \in \overline{\mathrm{I}}\left(u_{l}, u_{r}\right)$,

$$
\left|V\left(g_{k}, s_{1}, s_{2}\right)\right| \leq 2 \frac{C_{\mathrm{Lip}} C_{\max }}{m_{0}}\left(\left|g_{k}\right|+1\right) \leq 2 \frac{C_{\mathrm{Lip}} C_{\max }}{m_{0}}\left(\left|g_{l}\right|+C_{14}\left(C_{1}+1\right)\left|u_{r}-u_{l}\right|+1\right), \quad \forall k \in \llbracket 0, N \rrbracket,
$$

and the conclusion of the proof follows.

Thanks to the estimates provided by Lemma 3.1, we can now state the following property, from which theorem 2.6 follows.

Lemma 3.2 Under Hypotheses (7), let three reals $g_{l}, u_{l}, u_{r}$ be given. Let $\phi^{(N)}, \psi^{(N)}, \widehat{\mu}^{(N)}, \widehat{\nu}^{(N)}, \mu^{(N)}$, $\nu^{(N)}$ and $g^{(N)}$ for all $N \in \mathbb{N}$ with $N \geq 2$ be defined by (27)-(31). Then there exists a strictly increasing injection $\xi: \mathbb{N} \rightarrow \mathbb{N}$, such that the sequences $\left(\phi^{\xi(N)}\right)_{N \in \mathbb{N}},\left(\psi^{\xi(N)}\right)_{N \in \mathbb{N}},\left(\widehat{\mu}^{\xi(N)}\right)_{N \in \mathbb{N}},\left(\widehat{\nu}^{\xi(N)}\right)_{N \in \mathbb{N}}$, $\left(\mu^{\xi(N)}\right)_{N \in \mathbb{N}},\left(\nu^{\xi(N)}\right)_{N \in \mathbb{N}}$ and $\left(g^{\xi(N)}\right)_{N \in \mathbb{N}}$ converge in the following sense:

1. $\left(\phi^{\xi(N)}\right)_{N \in \mathbb{N}}$ (resp. $\left.\left(\psi^{\xi(N)}\right)_{N \in \mathbb{N}}\right)$ converge in $L^{1}\left(\overline{\mathrm{I}}\left(u_{l}, u_{r}\right)\right)$ to some functions $\phi \in L^{\infty}\left(\overline{\mathrm{I}}\left(u_{l}, u_{r}\right)\right) \cap$ $B V\left(\overline{\mathrm{I}}\left(u_{l}, u_{r}\right)\right)\left(\right.$ resp. $\left.\psi \in L^{\infty}\left(\overline{\mathrm{I}}\left(u_{l}, u_{r}\right)\right) \cap B V\left(\overline{\mathrm{I}}\left(u_{l}, u_{r}\right)\right)\right)$,

2. $\left(\widehat{\mu}^{\xi(N)}\right)_{N \in \mathbb{N}},\left(\widehat{\nu}^{\xi(N)}\right)_{N \in \mathbb{N}},\left(\mu^{\xi(N)}\right)_{N \in \mathbb{N}},\left(\nu^{\xi(N)}\right)_{N \in \mathbb{N}}$ and $\left(g^{\xi(N)}\right)_{N \in \mathbb{N}}$ respectively uniformly converge to some Lipschitz continuous functions $\widehat{\mu}, \widehat{\nu}, \mu, \nu$ and $g$, with $g\left(u_{l}\right)=g_{l}$, and $\mu(u)=-(c(u) g(u)+$ $d(u))$ and $\nu(f(u))=-(a(u) g(u)+b(u))$ for all $u \in \overline{\mathrm{I}}\left(u_{l}, u_{r}\right)$.

3. $\phi=\widehat{\mu}^{\prime}, \psi=\widehat{\nu}^{\prime}$ and $\psi(f(u))=\phi(u)$ for a.e. $u \in \overline{\mathrm{I}}\left(u_{l}, u_{r}\right)$.

4. if $u_{l} \geq u_{r}$ (resp. $\left.u_{l} \leq u_{r}\right)$, the function $\widehat{\mu}$ is the concave (resp. convex) hull of $\mu$ on $\overline{\mathrm{I}}\left(u_{l}, u_{r}\right)$ and the function $\widehat{\nu}$ is the convex (resp. concave) hull of $\nu$ on $\overline{\mathrm{I}}\left(f\left(u_{l}\right), f\left(u_{r}\right)\right)$.

\section{Proof.}

Proof of item 1

In the case $u_{r}<u_{l}$, we see that for all $N \geq 2$, the functions $\phi^{(N)}$ and $\psi^{(N)}$ are respectively non increasing and non decreasing, and bounded independently on $N$. Hence, thanks to Helly's theorem, we can extract a subsequence such that item 1 holds (the proof is similar in the case $u_{r}>u_{l}$ ).

Proof of item 2

We get from Lemma 3.1 that, for all $N \geq 2$, the functions $g^{(N)}$ are Lipschitz continuous with constants independent of $N$ and are bounded independently of $N$. Thanks to item 5 of lemma 3.1, we get that $\phi^{(N)}$ and $\psi^{(N)}$ are bounded independently of $N$, and the definition of $\widehat{\mu}$ and $\widehat{\nu}$ yields $\widehat{\mu}^{(N)^{\prime}}=\phi^{(N)}$ and $\widehat{\nu}^{(N)^{\prime}}=\psi^{(N)}$, which proves that these two functions are Lipschitz continuous on $\overline{\mathrm{I}}\left(u_{l}, u_{r}\right)$ with constants independent of $N$ and are bounded independently of $N$. We have, for all $j, k \in \llbracket 0, N \rrbracket$,

$$
\begin{aligned}
\left|\mu^{(N)}\left(u_{k}\right)-\mu^{(N)}\left(u_{j}\right)\right| & =\left|c\left(u_{j}\right) g_{j}-c\left(u_{k}\right) g_{k}+d\left(u_{j}\right)-d\left(u_{k}\right)\right| \\
& \leq \frac{1}{2}\left|c\left(u_{j}\right)-c\left(u_{k}\right)\right|\left|g_{k}+g_{j}\right|+\frac{1}{2}\left|c\left(u_{j}\right)+c\left(u_{k}\right)\right|\left|g_{j}-g_{k}\right|+\left|d\left(u_{j}\right)-d\left(u_{k}\right)\right| .
\end{aligned}
$$

From Lemma 3.1 and the above inequality, we easily deduce that $\mu^{(N)}$ is Lipschitz continuous on $\overline{\mathrm{I}}\left(u_{l}, u_{r}\right)$ with constant independent of $N$ and is bounded independently of $N$. The same conclusion clearly holds for $\nu^{(N)}$. 
We can therefore apply Ascoli's theorem, extracting a subsequence of that defined in the proof of item 1. Thanks to the definition of $\mu^{(N)}$ and $\nu^{(N)}$, we get that $\mu(u)=-(c(u) g(u)+d(u))$ and $\nu(f(u))=$ $-(a(u) g(u)+b(u))$ for all $u \in \overline{\mathrm{I}}\left(u_{l}, u_{r}\right)$.

Proof of item 3

This is an immediate consequence of (29).

Proof of item 4

Since the uniform limit of the convex (resp. concave) hull is the convex (resp. concave) hull of the uniform limit, this item is an immediate consequence of Lemma 3.1 and of item 2.

\section{Study of a finite volume scheme}

We now give a numerical scheme, which applies under Hypotheses (7) and (8) without restrictions. These hypotheses are therefore assumed in this section. Thanks to the proof of the convergence of the scheme given in this section (theorem 4.4), we then get the proof of Theorem 2.7.

Let $h>0$ be given, which will be called the space step in the following. We define a finite volume discretization of $\mathbb{R}$ by $K_{i}=(i h,(i+1) h)$, for all $i \in \mathbb{Z}$. Let $\delta t>0$ be given, which will be called the time step in the following. We set

$$
u_{i}^{(0)}=\frac{1}{h} \int_{i h}^{(i+1) h} u_{0}(x) \mathrm{d} x, \forall i \in \mathbb{Z} .
$$

Thanks to Hypothesis (8), the family $\left(u_{i}^{(0)}\right)_{i \in \mathbb{Z}}$ is such that there exists $i_{0}^{(0)} \in \mathbb{Z}$ such that, for all $i \in \mathbb{Z}$ with $i \leq i_{0}^{(0)}, u_{i}^{(0)}=\bar{u}_{0}$. We then define the finite volume scheme by induction. Let $n \in \mathbb{N}$ be given and let us assume that $\left(u_{i}^{(n)}\right)_{i \in \mathbb{Z}}$ is a given family of reals such that there exists $i_{0}^{(n)} \in \mathbb{Z}$ verifying

$$
u_{i}^{(n)}=\bar{u}_{0}, \forall i \in \mathbb{Z} \text { s.t. } i \leq i_{0}^{(n)} .
$$

We then define

$$
u_{i-\frac{1}{2}}^{(n)}=\bar{u}_{0}, v_{i-\frac{1}{2}}^{(n)}=\frac{1}{\delta t} \int_{n \delta t}^{(n+1) \delta t} \bar{v}_{0}(t) \mathrm{d} t, \quad \forall i \in \mathbb{Z} \text { s.t. } i \leq i_{0}^{(n)} .
$$

Let $i \geq i_{0}^{(n)}$. Let us assume that the values $v_{i-\frac{1}{2}}^{(n)}$ and $u_{i-\frac{1}{2}}^{(n)}$ are known. We define in the following the values $v_{i+\frac{1}{2}}^{(n)}$ and $u_{i+\frac{1}{2}}^{(n)}$, which permits to give the scheme by induction on $i \in \mathbb{Z}$. Let us define the function $\Phi_{i+\frac{1}{2}}^{(n)}: \mathbb{R} \times \mathbb{R} \rightarrow \mathbb{R}$ by

$$
\begin{aligned}
\Phi_{i+\frac{1}{2}}^{(n)}(u, v)= & f\left(u_{i}^{(n)}\right)+\frac{\delta t}{h}\left(a(u) v+b(u)-a\left(u_{i-\frac{1}{2}}^{(n)}\right) v_{i-\frac{1}{2}}^{(n)}-b\left(u_{i-\frac{1}{2}}^{(n)}\right)\right) \\
& -f\left(u_{i}^{(n)}+\frac{\delta t}{h}\left(c(u) v+d(u)-c\left(u_{i-\frac{1}{2}}^{(n)}\right) v_{i-\frac{1}{2}}^{(n)}-d\left(u_{i-\frac{1}{2}}^{(n)}\right)\right)\right) .
\end{aligned}
$$

We remark that this function $\Phi_{i+\frac{1}{2}}^{(n)}$ is locally Lipschitz continuous with respect to its arguments and verifies

$$
\partial_{2} \Phi_{i+\frac{1}{2}}^{(n)}(u, v)=\frac{\delta t}{h}\left(a(u)-c(u) f^{\prime}\left(\widehat{u}_{i}^{(n)}(u, v)\right)\right) \in\left[\frac{\delta t}{h} m_{0}, \frac{\delta t}{h} C_{\max }\left(1+C_{\text {Lip }}\right)\right], \quad \text { for a.e. } u, v \in \mathbb{R},
$$

with $\widehat{u}_{i}^{(n)}(u, v)=u_{i}^{(n)}+\frac{\delta t}{h}\left(c(u) v+d(u)-c\left(u_{i-\frac{1}{2}}^{(n)}\right) v_{i-\frac{1}{2}}^{(n)}-d\left(u_{i-\frac{1}{2}}^{(n)}\right)\right)$. Thus, for all $u \in \mathbb{R}$, the function $v \rightarrow \Phi_{i+\frac{1}{2}}^{(n)}(u, v)$ is Lipschitz continuous one-to-one from $\mathbb{R}$ to $\mathbb{R}$, and its reciprocal function is Lipschitz continuous from $\mathbb{R}$ to $\mathbb{R}$. Hence we implicitly define the Lipschitz continuous function $g_{i+\frac{1}{2}}^{(n)}$ by

$$
g_{i+\frac{1}{2}}^{(n)}: \mathbb{R} \rightarrow \mathbb{R}, u \mapsto v \text { s.t. } \Phi_{i+\frac{1}{2}}^{(n)}(u, v)=0 .
$$


Defining the Godunov flux $[10,9] F_{\mathrm{Go}}\left(\mu, s_{1}, s_{2}\right)$, for all $\mu \in C^{0}(\mathbb{R}, \mathbb{R})$ and for all $s_{1}, s_{2} \in \mathbb{R}$, by

$$
\begin{cases}\text { if } s_{1} \geq s_{2} & \text { then } F_{\mathrm{Go}}\left(\mu, s_{1}, s_{2}\right)=\max _{s \in\left[s_{2}, s_{1}\right]} \mu(s) \\ \text { else if } s_{1}<s_{2} & \text { then } F_{\mathrm{Go}}\left(\mu, s_{1}, s_{2}\right)=\min _{s \in\left[s_{1}, s_{2}\right]} \mu(s) .\end{cases}
$$

we define $u_{i+\frac{1}{2}}^{(n)} \in \mathbb{R}$ as a value associated with the Godunov scheme for the flux given by the function

$$
\mu_{i+\frac{1}{2}}^{(n)}: \mathbb{R} \rightarrow \mathbb{R}, u \mapsto-\left(c(u) g_{i+\frac{1}{2}}^{(n)}(u)+d(u)\right),
$$

the left value $u_{i}^{(n)}$ and the right value $u_{i+1}^{(n)}$, i.e.

$$
u_{i+\frac{1}{2}}^{(n)} \text { is any element of }\left\{s \in \overline{\mathrm{I}}\left(u_{i}^{(n)}, u_{i+1}^{(n)}\right), \mu_{i+\frac{1}{2}}^{(n)}(s)=F_{\mathrm{Go}}\left(\mu_{i+\frac{1}{2}}^{(n)}, u_{i}^{(n)}, u_{i+1}^{(n)}\right)\right\} .
$$

This allows to define the value $v_{i+\frac{1}{2}}^{(n)}$ by:

$$
v_{i+\frac{1}{2}}^{(n)}=g_{i+\frac{1}{2}}^{(n)}\left(u_{i+\frac{1}{2}}^{(n)}\right) .
$$

Relations (44)-(45) and the fact that $f$ is strictly decreasing imply that $f\left(u_{i+\frac{1}{2}}^{(n)}\right)$ reaches the Godunov scheme for the flux given by the function

$$
\nu_{i+\frac{1}{2}}^{(n)}: \mathbb{R} \rightarrow \mathbb{R}, w \mapsto-\left(a\left(f^{(-1)}(w)\right) g_{i+\frac{1}{2}}^{(n)}\left(f^{(-1)}(w)\right)+b\left(f^{(-1)}(w)\right)\right),
$$

the left value $f\left(u_{i}^{(n)}\right)$ and the right value $f\left(u_{i+1}^{(n)}\right)$, i.e.

$$
\nu_{i+\frac{1}{2}}^{(n)}\left(f\left(u_{i+\frac{1}{2}}^{(n)}\right)\right)=F_{\mathrm{Go}}\left(\nu_{i+\frac{1}{2}}^{(n)}, f\left(u_{i}^{(n)}\right), f\left(u_{i+1}^{(n)}\right)\right) .
$$

Hence we have defined values $u_{i+\frac{1}{2}}^{(n)}$ and $v_{i+\frac{1}{2}}^{(n)}$, and the induction on $i \in \mathbb{Z}$ with $i \geq i_{0}^{(n)}$, used in the definition of the scheme, is now complete. These values are such that

$$
\begin{aligned}
& f\left(u_{i}^{(n)}\right)+\frac{\delta t}{h}\left(a\left(u_{i+\frac{1}{2}}^{(n)}\right) v_{i+\frac{1}{2}}^{(n)}+b\left(u_{i+\frac{1}{2}}^{(n)}\right)-a\left(u_{i-\frac{1}{2}}^{(n)}\right) v_{i-\frac{1}{2}}^{(n)}-b\left(u_{i-\frac{1}{2}}^{(n)}\right)\right) \\
& =f\left(u_{i}^{(n)}+\frac{\delta t}{h}\left(c\left(u_{i+\frac{1}{2}}^{(n)}\right) v_{i+\frac{1}{2}}^{(n)}+d\left(u_{i+\frac{1}{2}}^{(n)}\right)-c\left(u_{i-\frac{1}{2}}^{(n)}\right) v_{i-\frac{1}{2}}^{(n)}-d\left(u_{i-\frac{1}{2}}^{(n)}\right)\right)\right) .
\end{aligned}
$$

We then set

$$
u_{i}^{(n+1)}=u_{i}^{(n)}+\frac{\delta t}{h}\left(c\left(u_{i+\frac{1}{2}}^{(n)}\right) v_{i+\frac{1}{2}}^{(n)}+d\left(u_{i+\frac{1}{2}}^{(n)}\right)-c\left(u_{i-\frac{1}{2}}^{(n)}\right) v_{i-\frac{1}{2}}^{(n)}-d\left(u_{i-\frac{1}{2}}^{(n)}\right)\right), \quad \forall i \in \mathbb{Z} .
$$

Note that, for all $i \in \mathbb{Z}$ with $i \leq i_{0}^{(n)}-1$, we get $u_{i}^{(n+1)}=\bar{u}_{0}$. We also denote by

$$
w_{i}^{(n)}=f\left(u_{i}^{(n)}\right), \forall i \in \mathbb{Z}, \forall n \in \mathbb{N} .
$$

Thanks to (52), we can write

$$
w_{i}^{(n+1)}=w_{i}^{(n)}+\frac{\delta t}{h}\left(a\left(u_{i+\frac{1}{2}}^{(n)}\right) v_{i+\frac{1}{2}}^{(n)}+b\left(u_{i+\frac{1}{2}}^{(n)}\right)-a\left(u_{i-\frac{1}{2}}^{(n)}\right) v_{i-\frac{1}{2}}^{(n)}-b\left(u_{i-\frac{1}{2}}^{(n)}\right)\right), \forall i \in \mathbb{Z} .
$$

We then see that the value

$$
i_{0}^{(n+1)}=i_{0}^{(n)}-1
$$


is such that (42) holds, replacing $n$ by $n+1$, which allows the definition of the scheme by induction on $n$ to hold.

Thanks to the definition of discrete values $u_{i}^{(n)}$ and $v_{i+\frac{1}{2}}^{(n)}$, for $n \in \mathbb{N}$ and $i \in \mathbb{Z}$, we can define the approximate functions $u_{h, \delta t}: \mathbb{R} \times \mathbb{R}_{+} \rightarrow \mathbb{R}$ and $v_{h, \delta t}: \mathbb{R}^{2} \times \mathbb{R}_{+} \rightarrow \mathbb{R}$ of $u$ and $v$ by

$$
\begin{aligned}
& u_{h, \delta t}(x, t)=u_{i}^{(n)}, \forall x \in(i h,(i+1) h) \text { and all } t \in[n \delta t,(n+1) \delta t), \forall i \in \mathbb{Z}, \forall n \in \mathbb{N}, \\
& v_{h, \delta t}(x, t)=v_{i-\frac{1}{2}}^{(n)}, \forall x \in\left(\left(i-\frac{1}{2}\right) h,\left(i+\frac{1}{2}\right) h\right) \text { and all } t \in[n \delta t,(n+1) \delta t), \forall i \in \mathbb{Z}, \forall n \in \mathbb{N} .
\end{aligned}
$$

We then have the following lemmas, which provides the estimates on the approximate solutions, used below in the convergence theorem.

Lemma 4.1 Assume that Hypotheses (7) and (8) hold. Let $h>0$ and $\delta t>0$ be given, and, for a given $n \in \mathbb{N}$, let $\left(u_{i}^{n}\right)_{i \in \mathbb{Z}}$ be a sequence of reals such that (42) holds for some $i_{0}^{(n)} \in \mathbb{Z}$ and such that

$$
\sum_{i \in \mathbb{Z}}\left|u_{i+1}^{(n)}-u_{i}^{(n)}\right| \leq W_{0}
$$

and

$$
U_{m} \leq u_{i}^{(n)} \leq U_{M}, \forall i \in \mathbb{Z}, \forall n \in \mathbb{N} .
$$

Let $\left(w_{i}^{n}\right)_{i \in \mathbb{Z}},\left(u_{i+\frac{1}{2}}^{n}\right)_{i \in \mathbb{Z}},\left(v_{i+\frac{1}{2}}^{n}\right)_{i \in \mathbb{Z}},\left(\mu_{i+\frac{1}{2}}^{n}\right)_{i \in \mathbb{Z}}$ and $\left(\nu_{i+\frac{1}{2}}^{(n)}\right)_{i \in \mathbb{Z}}$ be defined from the values $\left(u_{i}^{n}\right)_{i \in \mathbb{Z}}$ by (43) (55).

Then there exists $C_{3}, C_{4}, C_{5}, C_{6}$ and $C_{7}$, only depending on $\bar{V}_{0}, \bar{u}_{0}, U_{m}, U_{M}, W_{0}, C_{\max }, C_{\mathrm{Lip}}, f_{m}$, and $m_{0}$ such that

$$
\begin{gathered}
\left|v_{i+\frac{1}{2}}^{(n)}\right| \leq C_{3}, \quad \forall i \in \mathbb{Z}, \\
\left\|g_{i+\frac{1}{2}}^{(n)}\right\|_{L^{\infty}\left(U_{m}, U_{M}\right)} \leq C_{4}, \quad \forall i \in \mathbb{Z}, \\
\left\|\left(g_{i+\frac{1}{2}}^{(n)}\right)^{\prime}\right\|_{L^{\infty}\left(U_{m}, U_{M}\right)} \leq C_{5}, \quad \forall i \in \mathbb{Z}, \\
\left\|\left(\mu_{i+\frac{1}{2}}^{(n)}\right)^{\prime}\right\|_{L^{\infty}\left(U_{m}, U_{M}\right)} \leq C_{6}, \quad \forall i \in \mathbb{Z}, \\
\left\|\left(\nu_{i+\frac{1}{2}}^{(n)}\right)^{\prime}\right\|_{L^{\infty}\left(U_{m}, U_{M}\right)} \leq C_{7}, \quad \forall i \in \mathbb{Z} .
\end{gathered}
$$

Proof. For a given $i \in \mathbb{Z}$, we set

$$
\tau_{i, f}^{(n)}=\frac{f\left(u_{i}^{(n+1)}\right)-f\left(u_{i}^{(n)}\right)}{u_{i}^{(n+1)}-u_{i}^{(n)}} \text { if } u_{i}^{(n+1)} \neq u_{i}^{(n)} \text { else } \tau_{i, f}^{(n)}=-f_{m} .
$$

From (44)-(45) and definition (53) of $u_{i}^{n+1}$, we get

$$
v_{i+\frac{1}{2}}^{(n)}=\frac{\left(a\left(u_{i-\frac{1}{2}}^{(n)}\right)-c\left(u_{i-\frac{1}{2}}^{(n)}\right) \tau_{i, f}^{(n)}\right) v_{i-\frac{1}{2}}^{(n)}+b\left(u_{i-\frac{1}{2}}^{(n)}\right)-b\left(u_{i+\frac{1}{2}}^{(n)}\right)-\left(d\left(u_{i-\frac{1}{2}}^{(n)}\right)-d\left(u_{i+\frac{1}{2}}^{(n)}\right)\right) \tau_{i, f}^{(n)}}{a\left(u_{i+\frac{1}{2}}^{(n)}\right)-c\left(u_{i+\frac{1}{2}}^{(n)}\right) \tau_{i, f}^{(n)}} .
$$

Using again $C_{\text {Lip }}$ defined by (7), we define

$$
\tau_{i, a}^{(n)}=\frac{a\left(u_{i+\frac{1}{2}}^{(n)}\right)-a\left(u_{i-\frac{1}{2}}^{(n)}\right)}{u_{i+\frac{1}{2}}^{(n)}-u_{i-\frac{1}{2}}^{(n)}} \text { if } u_{i+\frac{1}{2}}^{(n)} \neq u_{i-\frac{1}{2}}^{(n)} \text { else } \tau_{i, a}^{(n)}=C_{\text {Lip }},
$$

and we define similarly $\tau_{i, b}^{(n)}, \tau_{i, c}^{(n)}$ and $\tau_{i, d}^{(n)}$, replacing respectively $a$ by $b, c$ and $d$. 
We can then write $v_{i+\frac{1}{2}}^{(n)}-v_{i-\frac{1}{2}}^{(n)}=\left(u_{i+\frac{1}{2}}^{(n)}-u_{i-\frac{1}{2}}^{(n)}\right)\left(G_{0} v_{i-\frac{1}{2}}^{(n)}+G_{1}\right)$ with

$$
G_{0}=\frac{\tau_{i, c}^{(n)} \tau_{i, f}^{(n)}-\tau_{i, a}^{(n)}}{a\left(u_{i+\frac{1}{2}}^{(n)}\right)-c\left(u_{i+\frac{1}{2}}^{(n)}\right) \tau_{i, f}^{(n)}} \text { and } G_{1}=\frac{\tau_{i, d}^{(n)} \tau_{i, f}^{(n)}-\tau_{i, b}^{(n)}}{a\left(u_{i+\frac{1}{2}}^{(n)}\right)-c\left(u_{i+\frac{1}{2}}^{(n)}\right) \tau_{i, f}^{(n)}},
$$

and therefore, since $a\left(u_{i+\frac{1}{2}}^{(n)}\right)-c\left(u_{i+\frac{1}{2}}^{(n)}\right) \tau_{i, f}^{(n)} \geq m_{0}$, we get $\left|G_{0}\right| \leq C_{8}$ and $\left|G_{1}\right| \leq C_{8}$ with $C_{8}=\frac{C_{\mathrm{Lip}}^{2}+C_{\mathrm{Lip}}}{m_{0}}$. We then have

$$
\left|v_{i+\frac{1}{2}}^{(n)}-v_{i-\frac{1}{2}}^{(n)}\right| \leq C_{8}\left|u_{i+\frac{1}{2}}^{(n)}-u_{i-\frac{1}{2}}^{(n)}\right|\left(\left|v_{i-\frac{1}{2}}^{(n)}\right|+1\right), \forall i \in \mathbb{Z} .
$$

We can the apply the discrete Gronwall lemma 5.3 (see appendix 2), starting from $i \geq i_{0}^{(n)}$. We thus obtain

$$
\forall i \in \mathbb{Z}, i \geq i_{0}^{(n)}\left|v_{i+\frac{1}{2}}^{(n)}\right| \leq\left(\left|\bar{V}_{0}\right|+1\right) \exp \left(C_{8} \sum_{j=i_{0}^{(n)}}^{i}\left|u_{j+\frac{1}{2}}^{n}-u_{j-\frac{1}{2}}^{n}\right|\right)-1 .
$$

Hence, thanks to (58) and (43), we have

$$
\left|v_{i+\frac{1}{2}}^{(n)}\right| \leq\left(\left|\bar{V}_{0}\right|+1\right) \exp \left(C_{8} W_{0}\right)-1, \forall i \in \mathbb{Z},
$$

which provides (60) with $C_{3}$ defined by the right hand side of the above inequality. From definition (44)-(45), setting $\widehat{u}_{i}^{(n)}: \mathbb{R} \rightarrow \mathbb{R}, s \mapsto u_{i}^{(n)}+\frac{\delta t}{h}\left(c(s) g_{i+\frac{1}{2}}^{(n)}(s)+d(s)-c\left(u_{i-\frac{1}{2}}^{(n)}\right) v_{i-\frac{1}{2}}^{(n)}-d\left(u_{i-\frac{1}{2}}^{(n)}\right)\right)$, we get by derivation, for a.e. $u \in \mathbb{R}$,

$$
\left(g_{i+\frac{1}{2}}^{(n)}\right)^{\prime}(u)=\frac{f^{\prime}\left(\widehat{u}_{i}^{(n)}(u)\right)\left(c^{\prime}(u) g_{i+\frac{1}{2}}^{(n)}(u)+d^{\prime}(u)\right)-a^{\prime}(u) g_{i+\frac{1}{2}}^{(n)}(u)-b^{\prime}(u)}{a(u)-c(u) f^{\prime}\left(\widehat{u}_{i}^{(n)}(u)\right)},
$$

which gives

$$
\left|\left(g_{i+\frac{1}{2}}^{(n)}\right)^{\prime}(u)\right| \leq C_{8}\left(\left|g_{i+\frac{1}{2}}^{(n)}(u)\right|+1\right) .
$$

We then apply Gronwall's lemma, starting from $u=u_{i+\frac{1}{2}}^{(n)} \in\left[U_{m}, U_{M}\right]$ thanks to (59). Since we have $\left|g_{i+\frac{1}{2}}^{(n)}\left(u_{i+\frac{1}{2}}^{(n)}\right)\right|=\left|v_{i+\frac{1}{2}}^{(n)}\right| \leq C_{3}$ by (60), we get (61) with $C_{4}=\left(C_{3}+1\right) \exp \left(C_{8}\left(U_{M}-U_{m}\right)\right)-1$. This therefore gives (62) with $C_{5}=C_{8}\left(C_{4}+1\right)$. We thus get (63), with $C_{6}=C_{\mathrm{Lip}} C_{4}+C_{\mathrm{max}} C_{5}+C_{\mathrm{Lip}}$ and (64) with $C_{7}=C_{6} / m_{0}$.

Lemma 4.2 Assume that Hypotheses (7) and (8) hold. Let $C_{9}$ be defined by the relation

$$
C_{9}=\frac{1}{2 \max \left(C_{6}, C_{7}\right)},
$$

where $C_{6}$ and $C_{7}$ are given by Lemma 4.1.

Then, for all $h>0$ and $\delta t>0$ be given such that

$$
\delta t \leq C_{9} h
$$

the values $\left(u_{i}^{n}\right)_{i \in \mathbb{Z}, n \in \mathbb{N}},\left(w_{i}^{n}\right)_{i \in \mathbb{Z}, n \in \mathbb{N}},\left(u_{i+\frac{1}{2}}^{n}\right)_{i \in \mathbb{Z}, n \in \mathbb{N}}$ and $\left(v_{i+\frac{1}{2}}^{n}\right)_{i \in \mathbb{Z}, n \in \mathbb{N}}$, defined by (41) - (55), satisfy

$$
\begin{gathered}
\sum_{i \in \mathbb{Z}}\left|u_{i+1}^{(n)}-u_{i}^{(n)}\right| \leq W_{0}, \quad \forall n \in \mathbb{N}, \\
U_{m} \leq u_{i}^{(n)} \leq U_{M}, \quad \forall i \in \mathbb{Z}, \forall n \in \mathbb{N} .
\end{gathered}
$$


Proof. We first remark that (67) and (68) hold for $n=0$ using definition (41). Let us assume that, for a given $n \in \mathbb{N}$, relations (67) and (68) hold. By the construction of the scheme, (42) holds, thus we can use the conclusions of Lemma 4.1. For a given $i \in \mathbb{Z}$, we define $\widetilde{u}_{i}^{(n+1)}$ by

$$
\widetilde{u}_{i}^{(n+1)}=u_{i}^{(n)}-\frac{\delta t}{h}\left(\mu_{i+\frac{1}{2}}^{(n)}\left(u_{i+\frac{1}{2}}^{(n)}\right)-\mu_{i+\frac{1}{2}}^{(n)}\left(u_{i}^{(n)}\right)+\mu_{i-\frac{1}{2}}^{(n)}\left(u_{i}^{(n)}\right)-\mu_{i-\frac{1}{2}}^{(n)}\left(u_{i-\frac{1}{2}}^{(n)}\right)\right) .
$$

Using again the notations $w_{i}^{(n)}=f\left(u_{i}^{(n)}\right)$ and $w_{i+\frac{1}{2}}^{(n)}=f\left(u_{i+\frac{1}{2}}^{(n)}\right)$ for all $i \in \mathbb{Z}$, we define $\widetilde{w}_{i}^{(n+1)}$, for all $i \in \mathbb{Z}$, by

$$
\widetilde{w}_{i}^{(n+1)}=w_{i}^{(n)}-\frac{\delta t}{h}\left(\nu_{i+\frac{1}{2}}^{(n)}\left(w_{i+\frac{1}{2}}^{(n)}\right)-\nu_{i+\frac{1}{2}}^{(n)}\left(w_{i}^{(n)}\right)+\nu_{i-\frac{1}{2}}^{(n)}\left(w_{i}^{(n)}\right)-\nu_{i-\frac{1}{2}}^{(n)}\left(w_{i-\frac{1}{2}}^{(n)}\right)\right) .
$$

Note that, in general, $\widetilde{w}_{i}^{(n+1)} \neq f\left(\widetilde{u}_{i}^{(n+1)}\right)$. We now define $\tau_{i,+}^{(n, u)}$ by

$$
\text { if } u_{i}^{(n)} \neq u_{i+\frac{1}{2}}^{(n)} \text {, then } \tau_{i,+}^{(n, u)}=\frac{\mu_{i+\frac{1}{2}}^{(n)}\left(u_{i}^{(n)}\right)-\mu_{i+\frac{1}{2}}^{(n)}\left(u_{i+\frac{1}{2}}^{(n)}\right)}{u_{i+\frac{1}{2}}^{(n)}-u_{i}^{(n)}} \text {, else } \tau_{i,+}^{(n, u)}=C_{6} \text {. }
$$

Note that, thanks to (48) and (63), we have $0 \leq \tau_{i,+}^{(n, u)} \leq C_{6}$. Similarly, we define $\tau_{i,-}^{(n, u)}$ by

$$
\text { if } u_{i}^{(n)} \neq u_{i-\frac{1}{2}}^{(n)} \text {, then } \tau_{i,-}^{(n, u)}=\frac{\mu_{i-\frac{1}{2}}^{(n)}\left(u_{i-\frac{1}{2}}^{(n)}\right)-\mu_{i-\frac{1}{2}}^{(n)}\left(u_{i}^{(n)}\right)}{u_{i-\frac{1}{2}}^{(n)}-u_{i}^{(n)}} \text {, else } \tau_{i,-}^{(n, u)}=C_{6}
$$

(again, thanks to (48) and (63), we have $0 \leq \tau_{i,-}^{(n, u)} \leq C_{6}$ ). We now define $\tau_{i,+}^{(n, w)}$ by

$$
\text { if } w_{i}^{(n)} \neq w_{i+\frac{1}{2}}^{(n)} \text {, then } \tau_{i,+}^{(n, w)}=\frac{\nu_{i+\frac{1}{2}}^{(n)}\left(w_{i}^{(n)}\right)-\nu_{i+\frac{1}{2}}^{(n)}\left(w_{i+\frac{1}{2}}^{(n)}\right)}{w_{i+\frac{1}{2}}^{(n)}-w_{i}^{(n)}} \text {, else } \tau_{i,+}^{(n, u)}=C_{7} \text {. }
$$

Note that, thanks to (51) and (64), we have $0 \leq \tau_{i,+}^{(n, w)} \leq C_{7}$. Similarly, we define $\tau_{i,-}^{(n, w)}$ by

$$
\text { if } w_{i}^{(n)} \neq w_{i-\frac{1}{2}}^{(n)} \text {, then } \tau_{i,-}^{(n, w)}=\frac{\nu_{i-\frac{1}{2}}^{(n)}\left(w_{i-\frac{1}{2}}^{(n)}\right)-\nu_{i-\frac{1}{2}}^{(n)}\left(w_{i}^{(n)}\right)}{w_{i-\frac{1}{2}}^{(n)}-w_{i}^{(n)}} \text {, else } \tau_{i,-}^{(n, w)}=C_{7}
$$

(again, thanks to $(51)$ and $(64)$, we have $0 \leq \tau_{i,-}^{(n, w)} \leq C_{7}$ ). We then get

$$
\widetilde{u}_{i}^{(n+1)}=u_{i}^{(n)}\left(1-\frac{\delta t}{h}\left(\tau_{i,+}^{(n, u)}+\tau_{i,-}^{(n, u)}\right)\right)+\frac{\delta t}{h} \tau_{i,+}^{(n, u)} u_{i+\frac{1}{2}}^{(n)}+\frac{\delta t}{h} \tau_{i,-}^{(n, u)} u_{i-\frac{1}{2}}^{(n)},
$$

and

$$
\widetilde{w}_{i}^{(n+1)}=w_{i}^{(n)}\left(1-\frac{\delta t}{h}\left(\tau_{i,+}^{(n, w)}+\tau_{i,-}^{(n, w)}\right)\right)+\frac{\delta t}{h} \tau_{i,+}^{(n, w)} w_{i+\frac{1}{2}}^{(n)}+\frac{\delta t}{h} \tau_{i,-}^{(n, w)} w_{i-\frac{1}{2}}^{(n)} .
$$

Defining $C_{9}$ by (65), we obtain from (69) that

$$
\widetilde{u}_{i}^{(n+1)} \in\left[\min \left(u_{i-\frac{1}{2}}^{(n)}, u_{i}^{(n)}, u_{i+\frac{1}{2}}^{(n)}\right), \max \left(u_{i-\frac{1}{2}}^{(n)}, u_{i}^{(n)}, u_{i+\frac{1}{2}}^{(n)}\right)\right]
$$

and from (70) that

$$
\begin{aligned}
\widetilde{w}_{i}^{(n+1)} \in & {\left[\min \left(w_{i-\frac{1}{2}}^{(n)}, w_{i}^{(n)}, w_{i+\frac{1}{2}}^{(n)}\right), \max \left(w_{i-\frac{1}{2}}^{(n)}, w_{i}^{(n)}, w_{i+\frac{1}{2}}^{(n)}\right)\right] } \\
& =\left[f\left(\max \left(u_{i-\frac{1}{2}}^{(n)}, u_{i}^{(n)}, u_{i+\frac{1}{2}}^{(n)}\right)\right), f\left(\min \left(u_{i-\frac{1}{2}}^{(n)}, u_{i}^{(n)}, u_{i+\frac{1}{2}}^{(n)}\right)\right)\right] .
\end{aligned}
$$


We now define the function $M_{i}^{(n)}:\left[U_{m}, U_{M}\right] \rightarrow \mathbb{R}, u \mapsto a\left(u_{i}^{(n)}\right) u-c\left(u_{i}^{(n)}\right) f(u)$. We then get from (71) and (72) that

$$
M_{i}^{(n)}\left(\min \left(u_{i-\frac{1}{2}}^{(n)}, u_{i}^{(n)}, u_{i+\frac{1}{2}}^{(n)}\right)\right) \leq a\left(u_{i}^{(n)}\right) \widetilde{u}_{i}^{(n+1)}-c\left(u_{i}^{(n)}\right) \widetilde{w}_{i}^{(n+1)} \leq M_{i}^{(n)}\left(\max \left(u_{i-\frac{1}{2}}^{(n)}, u_{i}^{(n)}, u_{i+\frac{1}{2}}^{(n)}\right)\right) .
$$

From the definition of $\widetilde{u}_{i}^{(n+1)}$ and $\widetilde{w}_{i}^{(n+1)}$ and from (53)-(55), we get

$$
M_{i}^{(n)}\left(u_{i}^{(n+1)}\right)=a\left(u_{i}^{(n)}\right) \widetilde{u}_{i}^{(n+1)}-c\left(u_{i}^{(n)}\right) \widetilde{w}_{i}^{(n+1)},
$$

since $a(u) \mu_{i+\frac{1}{2}}^{(n)}(u)-c(u) \nu_{i+\frac{1}{2}}^{(n)}(f(u))=a(u) \mu_{i-\frac{1}{2}}^{(n)}(u)-c(u) \nu_{i-\frac{1}{2}}^{(n)}(f(u))=-a(u) d(u)+c(u) b(u)$, for all $u \in\left[U_{m}, U_{M}\right]$. Thanks to (73) and (74), and to the strict monotony of the function $M_{i}^{(n)}$, we can write

$$
u_{i}^{(n+1)} \in\left[\min \left(u_{i-\frac{1}{2}}^{(n)}, u_{i}^{(n)}, u_{i+\frac{1}{2}}^{(n)}\right), \max \left(u_{i-\frac{1}{2}}^{(n)}, u_{i}^{(n)}, u_{i+\frac{1}{2}}^{(n)}\right)\right],
$$

which shows (68) for $n+1$. Thanks to the above property, we can now apply Lemma 5.4, which permits to conclude

$$
\sum_{i \in \mathbb{Z}}\left|u_{i}^{(n+1)}-u_{i+1}^{(n+1)}\right| \leq \sum_{i \in \mathbb{Z}}\left|u_{i}^{(n)}-u_{i+1}^{(n)}\right|
$$

which completes the proof of (67) for $n+1$.

Let us now classically deduce some bound for the variation in time, from that of the variation in space.

Lemma 4.3 Assume that Hypotheses (7) and (8) hold. Let $C_{9}$ be defined by Lemma 4.2 (thus only depending on $\bar{V}_{0}, \bar{u}_{0}, W_{0}, U_{m}, U_{M}, a, b, c, d$ and $\left.f\right)$. Let $h>0$ and $\delta t>0$ be given such that (66) holds. Then, there exists $C_{10}$, only depending on $\bar{V}_{0}, \bar{u}_{0}, W_{0}, U_{m}, U_{M}, a, b, c, d$ and $f$ such that the values $\left(u_{i}^{n}\right)_{i \in \mathbb{Z}, n \in \mathbb{N}},\left(w_{i}^{n}\right)_{i \in \mathbb{Z}, n \in \mathbb{N}},\left(u_{i+\frac{1}{2}}^{n}\right)_{i \in \mathbb{Z}, n \in \mathbb{N}}$ and $\left(v_{i+\frac{1}{2}}^{n}\right)_{i \in \mathbb{Z}, n \in \mathbb{N}}$, defined by (43) - (55), satisfy

$$
\sum_{n=\left\lfloor t_{1} / \delta t\right\rfloor}^{\left\lfloor t_{2} / \delta t\right\rfloor} \sum_{i \in \mathbb{Z}} h\left|u_{i}^{(n+1)}-u_{i}^{(n)}\right| \leq\left(t_{2}-t_{1}+\delta t\right) C_{10}, \forall t_{2} \geq t_{1} \geq 0,
$$

where, for all $s \in \mathbb{R}$, we denote by $\lfloor s\rfloor$ the biggest integer lower or equal to $s$.

Proof. We get, setting $u=u_{i-\frac{1}{2}}^{(n)}$ in (44)-(45), that $g_{i+\frac{1}{2}}^{(n)}\left(u_{i-\frac{1}{2}}^{(n)}\right)=g_{i-\frac{1}{2}}^{(n)}\left(u_{i-\frac{1}{2}}^{(n)}\right)$. Using (53), we get

$$
u_{i}^{(n+1)}=u_{i}^{(n)}+\frac{\delta t}{h}\left(c\left(u_{i+\frac{1}{2}}^{(n)}\right) g_{i+\frac{1}{2}}^{(n)}\left(u_{i+\frac{1}{2}}^{(n)}\right)+d\left(u_{i+\frac{1}{2}}^{(n)}\right)-c\left(u_{i-\frac{1}{2}}^{(n)}\right) g_{i+\frac{1}{2}}^{(n)}\left(u_{i-\frac{1}{2}}^{(n)}\right)-d\left(u_{i-\frac{1}{2}}^{(n)}\right)\right), \quad \forall i \in \mathbb{Z} .
$$

Using the Lipschitz constant $C_{6}$ defined in the proof of Lemma 4.2, this leads to

$$
\left|u_{i}^{(n+1)}-u_{i}^{(n)}\right| \leq \frac{\delta t}{h} C_{6}\left|u_{i+\frac{1}{2}}^{(n)}-u_{i-\frac{1}{2}}^{(n)}\right|, \forall i \in \mathbb{Z} .
$$

Therefore, we get

$$
\sum_{i \in \mathbb{Z}} h\left|u_{i}^{(n+1)}-u_{i}^{(n)}\right| \leq \delta t C_{6} W_{0}
$$

which provides (75) with $C_{10}=C_{6} W_{0}$.

It is now possible to state the convergence of the scheme. This is the aim of the following theorem.

Theorem 4.4 Assume that Hypotheses (7) and (8) hold. Let $C_{9}$ be defined by Lemma 4.2 (thus only depending on $\bar{V}_{0}, \bar{u}_{0}, W_{0}, U_{m}, U_{M}, a, b, c, d$ and $\left.f\right)$ and let $C_{11} \in\left(0, C_{9}\right)$ be given. Let $\left(h_{m}, \delta t_{m}\right)_{m \in \mathbb{N}}$ be a sequence of pairs of positive reals such that $\lim _{m \rightarrow \infty} h_{m}=0$ and such that $C_{11} h_{m} \leq \delta t_{m} \leq C_{9} h_{m}$ for all $m \in \mathbb{N}$. Then there exists a subsequence of $\left(h_{m}, \delta t_{m}\right)_{m \in \mathbb{N}}$, again denoted $\left(h_{m}, \delta t_{m}\right)_{m \in \mathbb{N}}$, such that the sequence of functions $\left(u_{h_{m}, \delta t_{m}}, v_{h_{m}, \delta t_{m}}\right)_{m \in \mathbb{N}}$ defined by (43) - (57) satisfies 
1. there exists $u \in L^{\infty}\left(\mathbb{R} \times \mathbb{R}_{+}\right)$with $u-u_{0} \in \operatorname{Lip}\left(\mathbb{R}_{+} ; L^{1}(\mathbb{R})\right) \cap L^{\infty}\left(\mathbb{R}_{+} ; B V(\mathbb{R})\right)$ such that

$$
\forall T \in \mathbb{R}_{+}, \lim _{m \rightarrow \infty} \sup _{t \in[0, T]}\left\|u_{h_{m}, \delta t_{m}}(\cdot, t)-u(\cdot, t)\right\|_{L^{1}(\mathbb{R})}=0,
$$

2. there exists $v \in L^{\infty}\left(\mathbb{R} \times \mathbb{R}_{+}\right)$such that $\left(v_{h_{m}, \delta t_{m}}\right)_{m \in \mathbb{N}}$ converges for the weak- $\star$ topology of $L^{\infty}\left(\mathbb{R} \times \mathbb{R}_{+}\right)$ to $v$,

3. this pair of functions $(u, v)$ is then an entropy weak solution of the system (9) in the sense of definition 2.3.

Proof. Let us examine the first item of the above theorem. It is easy to see that, for all $m \in \mathbb{N}$, $\left\|u_{h_{m}, \delta t_{m}}(\cdot, 0)-u_{0}\right\|_{L^{1}(\mathbb{R})} \leq h_{m} W_{0}$ thanks to (41). Thanks to Lemma 4.3, we get that, for all $t \in \mathbb{R}_{+}$, $\left\|u_{h_{m}, \delta t_{m}}(\cdot, t)-u_{0}\right\|_{L^{1}(\mathbb{R})} \leq h_{m} W_{0}+\left(t+\delta t_{m}\right) C_{10}$, which is bounded by $t C_{10}+C_{12}$ for all $m \in \mathbb{N}$. Thanks to Lemma 4.2 , we have $\left\|u_{h_{m}, \delta t_{m}}(\cdot, t)-u_{0}\right\|_{B V(\mathbb{R})} \leq 2 W_{0}$. Helly's theorem proves that the set $A(t)$ of all the functions $v \in L^{1}(\mathbb{R})$ such that $\|v\|_{L^{1}(\mathbb{R})} \leq t C_{10}+C_{12}$ and $\|v\|_{B V(\mathbb{R})} \leq 2 W_{0}$ is relatively compact in $L^{1}(\mathbb{R})$. We can then apply theorem 5.5 to the sequence $\left(u_{h_{m}, \delta t_{m}}-u_{0}\right)_{m \in \mathbb{N}}$, which allows to extract a sequence such that (76) holds.

Using (60), we again extract from this sequence a subsequence such that the second item holds.

Let us now prove the third item. Let $\varphi \in C_{c}^{\infty}\left(\mathbb{R} \times \mathbb{R}_{+}, \mathbb{R}\right)$ be given. Let $R>0$ and $T>0$ such that $\operatorname{support}(\varphi) \subset[-R, R] \times[0, T]$. We get (12), using (43), (56) and the hypothesis $C_{11} h_{m} \leq \delta t_{m}$, for all $m \in \mathbb{N}$ (this hypothesis is only needed here).

Let us now show (11). Let $\varphi \in C_{c}^{\infty}\left(\mathbb{R} \times \mathbb{R}_{+}, \mathbb{R}_{+}\right)$be given, and let $m \in \mathbb{N}$. For the simplicity of the notation, we omit some subscripts $m$ in the following calculations. For a given $n \in \mathbb{N}$, relations (53)-(55) read

$$
u_{i}^{(n+1)}=u_{i}^{(n)}-\frac{\delta t}{h}\left(F_{\mathrm{Go}}\left(\mu_{i+\frac{1}{2}}^{(n)}, u_{i}^{(n)}, u_{i+1}^{(n)}\right)-F_{\mathrm{Go}}\left(\mu_{i-\frac{1}{2}}^{(n)}, u_{i-1}^{(n)}, u_{i}^{(n)}\right)\right), \forall i \in \mathbb{Z},
$$

and

$$
f\left(u_{i}^{(n+1)}\right)=f\left(u_{i}^{(n)}\right)-\frac{\delta t}{h}\left(F_{\mathrm{Go}}\left(\nu_{i+\frac{1}{2}}^{(n)}, f\left(u_{i}^{(n)}\right), f\left(u_{i+1}^{(n)}\right)\right)-F_{\mathrm{Go}}\left(\nu_{i-\frac{1}{2}}^{(n)}, f\left(u_{i-1}^{(n)}\right), f\left(u_{i}^{(n)}\right)\right)\right), \forall i \in \mathbb{Z} .
$$

Let $\kappa \in \mathbb{R}$ be given. We get from the above relations, for all $i \in \mathbb{Z}$,

$$
\begin{aligned}
a(\kappa) u_{i}^{(n+1)}-c(\kappa) f\left(u_{i}^{(n+1)}\right)= & a(\kappa) u_{i}^{(n)}-c(\kappa) f\left(u_{i}^{(n)}\right) \\
& -a(\kappa) \frac{\delta t}{h}\left(F_{\mathrm{Go}}\left(\mu_{i+\frac{1}{2}}^{(n)}, u_{i}^{(n)}, u_{i+1}^{(n)}\right)-F_{\mathrm{Go}}\left(\mu_{i-\frac{1}{2}}^{(n)}, u_{i-1}^{(n)}, u_{i}^{(n)}\right)\right) \\
+ & c(\kappa) \frac{\delta t}{h}\left(F_{\mathrm{Go}}\left(\nu_{i+\frac{1}{2}}^{(n)}, f\left(u_{i}^{(n)}\right), f\left(u_{i+1}^{(n)}\right)\right)-F_{\mathrm{Go}}\left(\nu_{i-\frac{1}{2}}^{(n)}, f\left(u_{i-1}^{(n)}\right), f\left(u_{i}^{(n)}\right)\right)\right) .
\end{aligned}
$$

We thus have the relation $\Psi\left(u_{i}^{(n+1)}\right)=\Phi\left(u_{i-1}^{(n+1)}, u_{i}^{(n)}, u_{i+1}^{(n)}\right)$, where the functions $\Psi$ and $\Phi$ are such that, for all $s \in \mathbb{R}, \Psi(s)=a(\kappa) s-c(\kappa) f(s)$ and

$$
\begin{aligned}
\forall s_{1}, s_{2}, s_{3} \in \mathbb{R}, \Phi\left(s_{1}, s_{2}, s_{3}\right)= & a(\kappa) s_{2}-c(\kappa) f\left(s_{2}\right) \\
& -a(\kappa) \frac{\delta t}{h}\left(F_{\mathrm{Go}}\left(\mu_{i+\frac{1}{2}}^{(n)}, s_{2}, s_{3}\right)-F_{\mathrm{Go}}\left(\mu_{i-\frac{1}{2}}^{(n)}, s_{1}, s_{2}\right)\right) \\
+ & +c(\kappa) \frac{\delta t}{h}\left(F_{\mathrm{Go}}\left(\nu_{i+\frac{1}{2}}^{(n)}, f\left(s_{2}\right), f\left(s_{3}\right)\right)-F_{\mathrm{Go}}\left(\nu_{i-\frac{1}{2}}^{(n)}, f\left(s_{1}\right), f\left(s_{2}\right)\right)\right) .
\end{aligned}
$$

Thanks to the relation

$$
a(\kappa)\left(\mu_{i+\frac{1}{2}}^{(n)}(\kappa)-\mu_{i-\frac{1}{2}}^{(n)}(\kappa)\right)=c(\kappa)\left(\nu_{i+\frac{1}{2}}^{(n)}(f(\kappa))-\nu_{i-\frac{1}{2}}^{(n)}(f(\kappa))\right),
$$

we have $\Psi(\kappa)=\Phi(\kappa, \kappa, \kappa)$. We recall that, when $g \in C^{0}(\mathbb{R}, \mathbb{R})$, the function $F_{\mathrm{Go}}(g, \cdot, \cdot)$ defined by (46) is Lipschitz continuous and non decreasing with respect to its second argument, Lipschitz continuous and non increasing with respect to its third argument, with the same Lipschitz constants as $g$. We then see 
that $\Phi$ is non decreasing with respect to its first and third arguments in $\left[U_{m}, U_{M}\right]^{2}$. Thanks to condition (66) in which $C_{9}$ is given by (65), where $C_{6}$ and $C_{7}$ are respectively the Lipschitz constants of $\mu_{i \pm \frac{1}{2}}^{(n)}$ and $\nu_{i \pm \frac{1}{2}}^{(n)}$, we get that $\Phi$ is also non decreasing with respect to its second argument in $\left[U_{m}, U_{M}\right]$. Following a classical reasoning, we get that, if $\kappa \in\left[U_{m}, U_{M}\right]$, then

$$
\Psi\left(u_{i}^{(n+1)} \top \kappa\right) \leq \Phi\left(u_{i-1}^{(n)} \top \kappa, u_{i}^{(n)} \top \kappa, u_{i+1}^{(n)} \top \kappa\right) .
$$

This relation holds if $\kappa \geq U_{M}$ thanks to $\Psi(\kappa)=\Phi(\kappa, \kappa, \kappa)$, and it holds if $\kappa \leq U_{m}$ thanks to $\Psi\left(u_{i}^{(n+1)}\right)=$ $\Phi\left(u_{i-1}^{(n+1)}, u_{i}^{(n)}, u_{i+1}^{(n)}\right)$. Hence it holds in the general case of $\kappa \in \mathbb{R}$, and we can write $\Psi\left(u_{i}^{(n+1)} \top \kappa\right)-\Psi(\kappa) \leq$ $\Phi\left(u_{i-1}^{(n)} \top \kappa, u_{i}^{(n)} \top \kappa, u_{i+1}^{(n)} \top \kappa\right)-\Phi(\kappa, \kappa, \kappa)$. This gives

$$
\begin{aligned}
& \left.a(\kappa)\left(u_{i}^{(n+1)} \top \kappa-\kappa\right)-c(\kappa)\left(f\left(u_{i}^{(n+1)} \top \kappa\right)-f(\kappa)\right) \leq a(\kappa)\left(u_{i}^{(n)} \top \kappa-\kappa\right)-c(\kappa) f\left(u_{i}^{(n)} \top \kappa\right)-f(\kappa)\right) \\
& \quad-a(\kappa) \frac{\delta t}{h}\left(F_{\mathrm{Go}}\left(\mu_{i+\frac{1}{2}}^{(n)}, u_{i}^{(n)} \top \kappa, u_{i+1}^{(n)} \top \kappa\right)-F_{\mathrm{Go}}\left(\mu_{i-\frac{1}{2}}^{(n)}, u_{i-1}^{(n)} \top \kappa, u_{i}^{(n)} \top \kappa\right)\right) \\
& +c(\kappa) \frac{\delta t}{h}\left(F_{\mathrm{Go}}\left(\nu_{i+\frac{1}{2}}^{(n)}, f\left(u_{i}^{(n)} \top \kappa\right), f\left(u_{i+1}^{(n)} \top \kappa\right)\right)-F_{\mathrm{Go}}\left(\nu_{i-\frac{1}{2}}^{(n)}, f\left(u_{i-1}^{(n)} \top \kappa\right), f\left(u_{i}^{(n)} \top \kappa\right)\right)\right) .
\end{aligned}
$$

Thanks to the property $F_{\mathrm{Go}}(g, s, s)=g(s)$ for all $g \in C^{0}(\mathbb{R}, \mathbb{R})$ and $s \in \mathbb{R}$, we have

$$
\begin{aligned}
& a(\kappa)\left(F_{\mathrm{Go}}\left(\mu_{i+\frac{1}{2}}^{(n)}, s \top \kappa, s \top \kappa\right)-F_{\mathrm{Go}}\left(\mu_{i+\frac{1}{2}}^{(n)}, \kappa, \kappa\right)\right)- \\
& c(\kappa)\left(F_{\mathrm{Go}}\left(\nu_{i+\frac{1}{2}}^{(n)}, f(s \top \kappa), f(s \top \kappa)\right)-F_{\mathrm{Go}}\left(\nu_{i+\frac{1}{2}}^{(n)}, f(\kappa), f(\kappa)\right)\right)=-G_{0}(s) g_{i+\frac{1}{2}}^{(n)}(s)-G_{1}(s), \forall s \in \mathbb{R},
\end{aligned}
$$

where we define

$$
\begin{array}{ll}
G_{0}: \mathbb{R} \rightarrow \mathbb{R}, & s \mapsto a(\kappa) c(s \top \kappa)-c(\kappa) a(s \top \kappa), \\
G_{1}: \mathbb{R} \rightarrow \mathbb{R}, & s \mapsto a(\kappa)(d(s \top \kappa)-d(\kappa))-c(\kappa)(b(s \top \kappa)-b(\kappa)),
\end{array}
$$

where we remark that for all $s \in \mathbb{R},-G_{0}(s) g_{i+\frac{1}{2}}^{(n)}(s \top \kappa)-G_{1}(s)=-G_{0}(s) g_{i+\frac{1}{2}}^{(n)}(s)-G_{1}(s)$, since $G_{0}(s)=$ $G_{1}(s)=0$ for all $s<\kappa$. Note that the following relation holds:

$$
\begin{aligned}
& a(\kappa) F_{\mathrm{Go}}\left(\mu_{i+\frac{1}{2}}^{(n)}, \kappa, \kappa\right)-c(\kappa) F_{\mathrm{Go}}\left(\nu_{i+\frac{1}{2}}^{(n)}, f(\kappa), f(\kappa)\right)=-a(\kappa) d(\kappa)+c(\kappa) b(\kappa) \\
& =a(\kappa) F_{\mathrm{Go}}\left(\mu_{i-\frac{1}{2}}^{(n)}, \kappa, \kappa\right)-c(\kappa) F_{\mathrm{Go}}\left(\nu_{i-\frac{1}{2}}^{(n)}, f(\kappa), f(\kappa)\right) .
\end{aligned}
$$

We then get from $(77),(78)$ and (79)

$$
\begin{aligned}
& h\left(a(\kappa)\left(u_{i}^{(n+1)} \top \kappa-\kappa\right)-c(\kappa)\left(f\left(u_{i}^{(n+1)} \top \kappa\right)-f(\kappa)\right)-a(\kappa)\left(u_{i}^{(n)} \top \kappa-\kappa\right)+c(\kappa)\left(f\left(u_{i}^{(n)} \top \kappa\right)-f(\kappa)\right)\right) \\
& -\delta t\left(G_{0}\left(u_{i+\frac{1}{2}}^{(n)}\right) v_{i+\frac{1}{2}}^{(n)}+G_{1}\left(u_{i+\frac{1}{2}}^{(n)}\right)-G_{0}\left(u_{i-\frac{1}{2}}^{(n)}\right) v_{i-\frac{1}{2}}^{(n)}-G_{1}\left(u_{i-\frac{1}{2}}^{(n)}\right)\right) \leq \delta t\left(W_{i}^{n}-W_{i-1}^{(n)}\right),
\end{aligned}
$$

where we set

$$
\begin{aligned}
W_{i}^{n}= & -a(\kappa)\left(F_{\mathrm{Go}}\left(\mu_{i+\frac{1}{2}}^{(n)}, u_{i}^{(n)} \top \kappa, u_{i+1}^{(n)} \top \kappa\right)-F_{\mathrm{Go}}\left(\mu_{i+\frac{1}{2}}^{(n)}, u_{i+\frac{1}{2}}^{(n)} \top \kappa, u_{i+\frac{1}{2}}^{(n)} \top \kappa\right)\right) \\
& +c(\kappa)\left(F_{\mathrm{Go}}\left(\nu_{i+\frac{1}{2}}^{(n)}, f\left(u_{i}^{(n)} \top \kappa\right), f\left(u_{i+1}^{(n)} \top \kappa\right)\right)-F_{\mathrm{Go}}\left(\nu_{i+\frac{1}{2}}^{(n)}, f\left(u_{i+\frac{1}{2}}^{(n)} \top \kappa\right), f\left(u_{i+\frac{1}{2}}^{(n)} \top \kappa\right)\right)\right) .
\end{aligned}
$$

We then multiply (80) by $\varphi\left(\left(i+\frac{1}{2}\right) h, n \delta t\right)$. Gathering the terms obtained by summing the result on $n \in \mathbb{N}$ and $i \in \mathbb{Z}$, we then obtain $T_{1}^{(m)}+T_{2}^{(m)}+T_{3}^{(m)} \leq T_{4}^{(m)}$, with

$$
\begin{gathered}
T_{1}^{(m)}=-\sum_{n \in \mathbb{N}^{\star}} \sum_{i \in \mathbb{Z}} h\left(a(\kappa)\left(u_{i}^{(n)} \top \kappa-\kappa\right)-c(\kappa)\left(f\left(u_{i}^{(n)} \top \kappa\right)-f(\kappa)\right)\right)\left(\varphi\left(\left(i+\frac{1}{2}\right) h, n \delta t\right)-\varphi\left(\left(i+\frac{1}{2}\right) h,(n-1) \delta t\right),\right. \\
T_{2}^{(m)}=-\sum_{i \in \mathbb{Z}} h\left(a(\kappa)\left(u_{i}^{(0)} \top \kappa-\kappa\right)-c(\kappa)\left(f\left(u_{i}^{(0)} \top \kappa\right)-f(\kappa)\right)\right) \varphi\left(\left(i+\frac{1}{2}\right) h, 0\right),
\end{gathered}
$$




$$
T_{3}^{(m)}=\sum_{n \in \mathbb{N}^{\star}} \delta t \sum_{i \in \mathbb{Z}}\left(G_{0}\left(u_{i-\frac{1}{2}}^{(n)}\right) v_{i-\frac{1}{2}}^{(n)}+G_{1}\left(u_{i-\frac{1}{2}}^{(n)}\right)\right)\left(\varphi\left(\left(i+\frac{1}{2}\right) h, n \delta t\right)-\varphi\left(\left(i-\frac{1}{2}\right) h, n \delta t\right)\right)
$$

and

$$
T_{4}^{(m)}=\sum_{n \in \mathbb{N}^{\star}} \delta t \sum_{i \in \mathbb{Z}} W_{i-1}^{(n)}\left(\varphi\left(\left(i-\frac{1}{2}\right) h, n \delta t\right)-\varphi\left(\left(i+\frac{1}{2}\right) h, n \delta t\right)\right)
$$

We then classically obtain that

$$
\begin{gathered}
\lim _{m \rightarrow \infty} T_{1}^{(m)}=-\int_{\mathbb{R}_{+}} \int_{\mathbb{R}}(a(\kappa)(u(x, t) \top \kappa-\kappa)-c(\kappa)(f(u(x, t) \top \kappa)-f(\kappa))) \varphi_{t}(x, t) \mathrm{d} x \mathrm{~d} t, \\
\lim _{m \rightarrow \infty} T_{2}^{(m)}=-\int_{\mathbb{R}}\left(a(\kappa)\left(u_{0}(x) \top \kappa-\kappa\right)-c(\kappa)\left(f\left(u_{0}(x) \top \kappa\right)-f(\kappa)\right)\right) \varphi(x, 0) \mathrm{d} x \mathrm{~d} t,
\end{gathered}
$$

and, thanks to (67), we get

$$
\lim _{m \rightarrow \infty} T_{3}^{(m)}=\int_{\mathbb{R}_{+}} \int_{\mathbb{R}}\left(G_{0}(u(x, t)) v(x, t)+G_{1}(u(x, t))\right) \varphi_{x}(x, t) \mathrm{d} x \mathrm{~d} t .
$$

Turning to the study of $T_{4}^{(m)}$, we get, from the Lipschitz continuity properties of $F_{\text {Go }}$ and using $u_{i+\frac{1}{2}}^{(n)} \in$ $\overline{\mathrm{I}}\left(u_{i}^{(n)}, u_{i+1}^{(n)}\right)$,

$$
\left|W_{i}^{n}\right| \leq C_{\max }\left(C_{6}+C_{7} C_{\text {Lip }}\right)\left|u_{i+1}^{(n)}-u_{i}^{(n)}\right|
$$

Thanks to the above inequalities, we obtain that

$$
\left|T_{4}^{(m)}\right| \leq C_{\max }\left(C_{6}+C_{7} C_{\mathrm{Lip}}\right) \sum_{n \in \mathbb{N}^{\star}} \delta \sum_{i \in \mathbb{Z}}\left|u_{i-1}^{(n)}-u_{i}^{(n)}\right|\left|\varphi\left(\left(i-\frac{1}{2}\right) h, n \delta t\right)-\varphi\left(\left(i+\frac{1}{2}\right) h, n \delta t\right)\right|
$$

which yields, setting $C_{13}=\left\|\partial_{1} \varphi\right\|_{L^{\infty}\left(\mathbb{R} \times \mathbb{R}_{+}\right)} C_{\max }\left(C_{6}+C_{7} C_{\text {Lip }}\right)$,

$$
\left|T_{4}^{(m)}\right| \leq h C_{13} \sum_{n=0}^{\lfloor T / \delta t\rfloor} \delta t \sum_{i \in \mathbb{Z}}\left|u_{i-1}^{(n)}-u_{i}^{(n)}\right| .
$$

This gives, using (67) and reintroducing subscripts $m,\left|T_{4}^{(m)}\right| \leq h_{m}\left(T+\delta t_{m}\right) C_{13} W_{0}$ and finally $\lim _{m \rightarrow \infty} T_{4}^{(m)}=$ 0 , which concludes the proof of the first inequality of (11). The second inequality is proven exactly in the same way.

The proof of (10) is similar and simpler. For a given $\varphi \in C_{c}^{\infty}\left(\mathbb{R} \times \mathbb{R}_{+}, \mathbb{R}\right)$ and $m \in \mathbb{N}$, we multiply (53) and (55) by $h \varphi\left(\left(i+\frac{1}{2}\right) h_{m}, n \delta t_{m}\right)$, and we sum the result over $i \in \mathbb{Z}$ and $n \in \mathbb{N}$. We then pass to the limit $m \rightarrow \infty$, again using the convergence of $\left(u_{h_{m}, \delta t_{m}}\right)_{m \in \mathbb{N}}$ to $u$ in $L^{1}([-R, R] \times[0, T])$, the convergence of $\left(v_{h_{m}, \delta t_{m}}\right)_{m \in \mathbb{N}}$ for the weak- $\star$ topology of $L^{\infty}\left(\mathbb{R} \times \mathbb{R}_{+}\right)$to $v$, the estimate (67), and following the same reasoning as above. We then get that (10) is satisfied by $(u, v)$. This concludes the proof that $(u, v)$ is an entropy weak solution of the system (9) in the sense of definition 2.3.

\section{$5 \quad$ Numerical results}

We provide in this section some numerical results, obtained with the generalized Godunov scheme presented in section 4 . We consider the data of the third analytical example in section 2, corresponding to the simulation of a bubble initially present in the domain. The value for $C_{9}$ provided by (65) is not sharp enough to be used for practically setting the value of the time step as a function of the space step. Hence, using the analytical values taken by the function $v$, it was possible to assess a much more accurate value 
for $\delta t / h=\overline{C_{9}}$, ensuring the stability of the scheme. We have thus taken $\delta t=10^{-7}$ for $h=10^{-4}$. We show on figures 3 and 4 respectively the functions $u_{h, \delta t}\left(x, t_{0}\right)$ and $v_{h, \delta t}\left(x, t_{0}\right)$ at time $t_{0}=0.1$.

Let us first observe that the numerical solution is very close to the analytical one shown in figures 1-2. Classically, we note that the contact discontinuity is more subject to numerical diffusion than the shocks. We remark that the approximate solution $v_{h, \delta t}$ shows monotony properties with respect to $x$, but presents periodic oscillations with respect to $t$ (see figure (5)). The period of these oscillations only depends on the space step, and numerically corresponds to the time needed for dissolving the gaseous component into the water phase at the maximum concentration in one control volume, starting with pure water (this time is also that of the apparition of the gaseous phase in this control volume). This is in agreement with the theoretical convergence properties in section 4 , only based on the proof of the convergence of $v_{h, \delta t}$ to $v$ for the weak- $\star$ convergence of $L^{\infty}\left(\mathbb{R} \times \mathbb{R}_{+}\right)$. Indeed, it is easy to see that some $B V$ estimate could be considered for $v$ with respect to the space variable, but the lack of term $v_{t}$ prevents from obtaining a similar estimate with respect to the time variable. However, a time average of $v_{h, \delta t}(x, t)$ can be shown to accurately converge to the analytical value obtained in section 2 . Let us compute the numerical error with respect to the analytical solution given in section 2 . Since in this case, we have $u_{h, \delta t}(x, t)=u(x, t)$ for sufficiently large and small values of $x$, we can compute

$$
E(h, \delta t, t)=\int_{\mathbb{R}}\left|u(x, t)-u_{h, \delta t}(x, t)\right| \mathrm{d} x .
$$

We then provide $e(h)=E\left(h, \overline{C_{9}} h, 0.1\right)$ as a function of $h$ in figure 6 , showing a numerical convergence order about 0.5 .

\section{Appendix: some technical results}

Lemma 5.1 (Lipschitz continuity of $G$ ) Assuming Hypotheses (7), let $G$ be the function defined by (25)-(26). Then there exists $C_{14}$, which only depends on $C_{\mathrm{Lip}}, C_{\max }, m_{0}$ and $f_{m}$, such that

$$
\forall s_{1}, s_{2}, s_{3}, g_{1} \in \mathbb{R},\left\{\begin{array}{l}
\left|G\left(g_{1}, s_{1}, s_{2}\right)-G\left(g_{1}, s_{1}, s_{3}\right)\right| \leq C_{14}\left(\left|g_{1}\right|+1\right)\left|s_{3}-s_{2}\right|, \\
\left|G\left(g_{1}, s_{1}, s_{2}\right)-G\left(g_{1}, s_{3}, s_{2}\right)\right| \leq C_{14}\left(\left|g_{1}\right|+1\right)\left|s_{3}-s_{1}\right| .
\end{array}\right.
$$

Proof. Prolonging $\tau_{f}\left(s_{1}, s_{2}\right)$ by $\tau_{f}\left(s_{1}, s_{2}\right)=-C_{\text {Lip }}$ for $s_{1}=s_{2}$, and using (25)-(26), we can write

$$
\forall s_{1}, s_{2}, g_{1} \in \mathbb{R}, G\left(g_{1}, s_{1}, s_{2}\right)=g_{1}+H_{0}\left(s_{1}, s_{2}\right) g_{1}+H_{1}\left(s_{1}, s_{2}\right),
$$

with

$$
\begin{aligned}
\forall\left(s_{1}, s_{2}\right) \in \mathbb{R}^{2}, \quad H_{0}\left(s_{1}, s_{2}\right) & =\frac{\left(c\left(s_{2}\right)-c\left(s_{1}\right)\right) \tau_{f}\left(s_{1}, s_{2}\right)-\left(a\left(s_{2}\right)-a\left(s_{1}\right)\right)}{a\left(s_{2}\right)-c\left(s_{2}\right) \tau_{f}\left(s_{1}, s_{2}\right)}, \\
H_{1}\left(s_{1}, s_{2}\right) & =\frac{\left(d\left(s_{2}\right)-d\left(s_{1}\right)\right) \tau_{f}\left(s_{1}, s_{2}\right)-\left(b\left(s_{2}\right)-b\left(s_{1}\right)\right)}{a\left(s_{2}\right)-c\left(s_{2}\right) \tau_{f}\left(s_{1}, s_{2}\right)} .
\end{aligned}
$$

Let us prove that the functions $H_{0}$ and $H_{1}$ are Lipschitz continuous on $\mathbb{R}^{2}$, which immediately gives (83) from (84). Let us first remark that these functions are clearly continuous in all $\left(s_{1}, s_{2}\right) \in \mathbb{R}^{2}$, with $s_{1} \neq s_{2}$. We now remark that, for all $\left(s_{1}, s_{2}\right) \in \mathbb{R}^{2}$, with $s_{1} \neq s_{2}$, we have

$$
\frac{H_{0}\left(s_{1}, s_{2}\right)}{s_{2}-s_{1}}=\frac{\tau_{c}\left(s_{1}, s_{2}\right) \tau_{f}\left(s_{1}, s_{2}\right)-\tau_{a}\left(s_{1}, s_{2}\right)}{a\left(s_{2}\right)-c\left(s_{2}\right) \tau_{f}\left(s_{1}, s_{2}\right)}
$$

which shows that

$$
\forall\left(s_{1}, s_{2}\right) \in \mathbb{R}^{2},\left|H_{0}\left(s_{1}, s_{2}\right)\right| \leq\left|s_{2}-s_{1}\right| \frac{C_{\text {Lip }}^{2}+C_{\text {Lip }}}{m_{0}} .
$$

The above relation shows that $H_{0}$ is continuous on $\mathbb{R}^{2}$. The same conclusion holds for $H_{1}$. It now suffices to show that the partial derivatives of $H_{0}$ and $H_{1}$ are bounded almost everywhere. Let $\left(s_{1}, s_{2}\right) \in \mathbb{R}^{2}$ with $s_{1} \neq s_{2}$. We can write $H_{0}\left(s_{1}, s_{2}\right)=\widehat{H}_{0}\left(s_{1}, s_{2}, \tau_{f}\left(s_{1}, s_{2}\right)\right)$, with

$$
\forall\left(s_{1}, s_{2}\right) \in \mathbb{R}^{2}, \forall t \in\left[-C_{\text {Lip }},-f_{m}\right], \widehat{H}_{0}\left(s_{1}, s_{2}, t\right)=\frac{\left(c\left(s_{2}\right)-c\left(s_{1}\right)\right) t-\left(a\left(s_{2}\right)-a\left(s_{1}\right)\right)}{a\left(s_{2}\right)-c\left(s_{2}\right) t} .
$$


We then easily get that, for a.e. $\left(s_{1}, s_{2}\right) \in \mathbb{R}^{2}$ and $t \in\left[-C_{\text {Lip }},-f_{m}\right]$,

$$
\begin{gathered}
\left|\partial_{1} \widehat{H}_{0}\left(s_{1}, s_{2}, t\right)\right| \leq \frac{C_{\mathrm{Lip}}+C_{\mathrm{Lip}}^{2}}{m_{0}}, \\
\left|\partial_{2} \widehat{H}_{0}\left(s_{1}, s_{2}, t\right)\right| \leq \frac{\left(C_{\mathrm{Lip}}+C_{\mathrm{Lip}}^{2}\right)\left(C_{\mathrm{max}}+C_{\mathrm{Lip}} C_{\mathrm{max}}\right)}{m_{0}^{2}} \\
\left|\partial_{3} \widehat{H}_{0}\left(s_{1}, s_{2}, t\right)\right| \leq\left|s_{2}-s_{1}\right|\left(\frac{C_{\mathrm{Lip}}+C_{\mathrm{Lip}}^{2}}{m_{0}}+\frac{C_{\max }\left(C_{\mathrm{Lip}}+C_{\mathrm{Lip}}^{2}\right)}{m_{0}^{2}}\right) .
\end{gathered}
$$

Since we get that

$$
\text { for a.e. }\left(s_{1}, s_{2}\right) \in \mathbb{R}^{2}, \forall i=1,2,\left|\partial_{i} \tau_{f}\left(s_{1}, s_{2}\right)\right| \leq \frac{2 C_{\text {Lip }}}{\left|s_{2}-s_{1}\right|},
$$

we conclude that $H_{0}$ is Lipschitz continuous on $\mathbb{R}^{2}$, since we have

$$
\begin{aligned}
& \text { for a.e. }\left(s_{1}, s_{2}\right) \in \mathbb{R}^{2}, \forall i=1,2, \\
& \partial_{i} H_{0}\left(s_{1}, s_{2}\right)=\partial_{i} \widehat{H}_{0}\left(s_{1}, s_{2}, \tau_{f}\left(s_{1}, s_{2}\right)\right)+\partial_{i} \tau_{f}\left(s_{1}, s_{2}\right) \partial_{3} \widehat{H}_{0}\left(s_{1}, s_{2}, \tau_{f}\left(s_{1}, s_{2}\right)\right) .
\end{aligned}
$$

We proceed exactly in the same way for $H_{1}$, getting the same conclusion.

Lemma 5.2 (Monotony property of $V$ ) Under Hypotheses (7), let $V$ and $G$ be the functions defined by (25)-(26). Then

$$
\begin{aligned}
& \forall u_{1}, u_{2}, u_{3}, g_{1} \in \mathbb{R},\left(u_{1}-u_{2}\right)\left(u_{2}-u_{3}\right)>0 \Rightarrow \\
& \exists \alpha>0,\left(V\left(g_{1}, u_{1}, u_{3}\right)-V\left(g_{1}, u_{1}, u_{2}\right)\right)=\alpha\left(V\left(G\left(g_{1}, u_{1}, u_{2}\right), u_{2}, u_{3}\right)-V\left(g_{1}, u_{1}, u_{3}\right)\right) .
\end{aligned}
$$

Proof. Let $u_{1}, u_{2}, u_{3}, g_{1} \in \mathbb{R}$ be such that $\left(u_{1}-u_{2}\right)\left(u_{2}-u_{3}\right)>0$. Let us denote by $g_{2}=G\left(g_{1}, u_{1}, u_{2}\right)$, $v_{2}=V\left(g_{1}, u_{1}, u_{2}\right), g_{3}=G\left(g_{1}, u_{1}, u_{3}\right), v_{3}=V\left(g_{1}, u_{1}, u_{3}\right)$ and $g_{3}^{\prime}=G\left(g_{2}, u_{2}, u_{3}\right), v_{3}^{\prime}=V\left(g_{2}, u_{2}, u_{3}\right)$. Using (24), we can write

$$
\begin{array}{ll}
v_{2}\left(f\left(u_{1}\right)-f\left(u_{2}\right)\right) & =-\left(a\left(u_{1}\right) g_{1}+b\left(u_{1}\right)-a\left(u_{2}\right) g_{2}-b\left(u_{2}\right)\right) \\
v_{2}\left(u_{1}-u_{2}\right) & =-\left(c\left(u_{1}\right) g_{1}+d\left(u_{1}\right)-c\left(u_{2}\right) g_{2}-d\left(u_{2}\right)\right), \\
v_{3}^{\prime}\left(f\left(u_{2}\right)-f\left(u_{3}\right)\right) & =-\left(a\left(u_{2}\right) g_{2}+b\left(u_{2}\right)-a\left(u_{3}\right) g_{3}^{\prime}-b\left(u_{3}\right)\right) \\
v_{3}^{\prime}\left(u_{2}-u_{3}\right) & =-\left(c\left(u_{2}\right) g_{2}+d\left(u_{2}\right)-c\left(u_{3}\right) g_{3}^{\prime}-d\left(u_{3}\right)\right) . \\
v_{3}\left(f\left(u_{1}\right)-f\left(u_{3}\right)\right) & =-\left(a\left(u_{1}\right) g_{1}+b\left(u_{1}\right)-a\left(u_{3}\right) g_{3}-b\left(u_{3}\right)\right) \\
v_{3}\left(u_{1}-u_{3}\right) & =-\left(c\left(u_{1}\right) g_{1}+d\left(u_{1}\right)-c\left(u_{3}\right) g_{3}-d\left(u_{3}\right)\right),
\end{array}
$$

This provides, by addition of the first and second system, elimination of $g_{3}^{\prime}$, and by elimination of $g_{3}$ in the third system,

$$
\begin{aligned}
& v_{3}^{\prime}\left(c\left(u_{3}\right)\left(f\left(u_{2}\right)-f\left(u_{3}\right)\right)-a\left(u_{3}\right)\left(u_{2}-u_{3}\right)\right)+v_{2}\left(c\left(u_{3}\right)\left(f\left(u_{1}\right)-f\left(u_{2}\right)\right)-a\left(u_{3}\right)\left(u_{1}-u_{2}\right)\right)= \\
& v_{3}\left(c\left(u_{3}\right)\left(f\left(u_{1}\right)-f\left(u_{3}\right)\right)-a\left(u_{3}\right)\left(u_{1}-u_{3}\right)\right) .
\end{aligned}
$$

We thus get

$$
\begin{aligned}
& \left(v_{3}^{\prime}-v_{3}\right)\left(c\left(u_{3}\right)\left(f\left(u_{2}\right)-f\left(u_{3}\right)\right)-a\left(u_{3}\right)\left(u_{2}-u_{3}\right)\right)= \\
& \left(v_{3}-v_{2}\right)\left(c\left(u_{3}\right)\left(f\left(u_{1}\right)-f\left(u_{2}\right)\right)-a\left(u_{3}\right)\left(u_{1}-u_{2}\right)\right),
\end{aligned}
$$

which gives (85) thanks to Hypotheses (7).

Lemma 5.3 (Discrete Gronwall's lemma) Let $N \in \mathbb{N}^{\star}$ be given, and let $\left(g_{k}\right)_{k \in \llbracket 0, N \rrbracket}$ and $\left(u_{k}\right)_{k \in \llbracket 0, N \rrbracket}$ be discrete sequences of reals, such that there exists $C_{G} \geq 0$ with

$$
\left|g_{k+1}-g_{k}\right| \leq C_{G}\left(\left|g_{k}\right|+1\right)\left|u_{k+1}-u_{k}\right|, \forall k \in \llbracket 0, N-1 \rrbracket .
$$

Then the following holds

$$
\left|g_{k}\right| \leq\left(\left|g_{0}\right|+1\right) \exp \left[C_{G} \sum_{i=0}^{N-1}\left|u_{i+1}-u_{i}\right|\right]-1, \quad \forall k \in \llbracket 0, N \rrbracket .
$$


Proof. Let $k \in \llbracket 0, N-1 \rrbracket$. From (86), we get,

$$
\left|g_{k+1}\right|-\left|g_{k}\right| \leq C_{G}\left(\left|g_{k}\right|+1\right)\left|u_{k+1}-u_{k}\right|,
$$

which gives

$$
\left|g_{k+1}\right| \leq\left|g_{k}\right|\left(1+C_{G}\left|u_{k+1}-u_{k}\right|\right)+C_{G}\left|u_{k+1}-u_{k}\right|,
$$

and therefore

$$
\left|g_{k+1}\right|+1 \leq\left(\left|g_{k}\right|+1\right)\left(1+C_{G}\left|u_{k+1}-u_{k}\right|\right) .
$$

Since the above inequality holds for all $k \in \llbracket 0, N-1 \rrbracket$, we get

$$
\left|g_{k+1}\right|+1 \leq\left(\left|g_{0}\right|+1\right) \prod_{i=0}^{k}\left(1+C_{G}\left|u_{i+1}-u_{i}\right|\right) .
$$

Hence we obtain,

$$
\log \left[\left|g_{k+1}\right|+1\right] \leq \log \left(\left|g_{0}\right|+1\right)+\sum_{i=0}^{k} \log \left(1+C_{G}\left|u_{i+1}-u_{i}\right|\right) .
$$

Since $\forall s \geq 0, \log (1+s) \leq s$, we have

$$
\log \left[\left|g_{k+1}\right|+1\right] \leq \log \left(\left|g_{0}\right|+1\right)+\sum_{i=0}^{k} C_{G}\left|u_{i+1}-u_{i}\right| .
$$

Therefore, using $\sum_{i=0}^{k}\left|u_{i+1}-u_{i}\right| \leq \sum_{i=0}^{N-1}\left|u_{i+1}-u_{i}\right|$, we get

$$
\left|g_{k+1}\right|+1 \leq\left(\left|g_{0}\right|+1\right) \exp \left[C_{G} \sum_{i=0}^{N-1}\left|u_{i+1}-u_{i}\right|\right] .
$$

The conclusion of the proof follows.

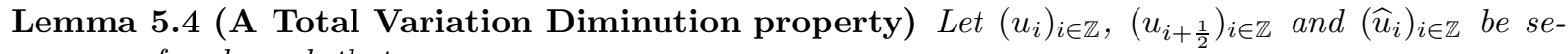
quences of reals such that

1. $\sum_{i \in \mathbb{Z}}\left|u_{i+1}-u_{i}\right|<\infty$,

2. for all $i \in \mathbb{Z}, u_{i+\frac{1}{2}} \in \overline{\mathrm{I}}\left(u_{i}, u_{i+1}\right)$,

3. for all $i \in \mathbb{Z}, \widehat{u}_{i} \in\left[\min \left(u_{i-\frac{1}{2}}, u_{i}, u_{i+\frac{1}{2}}\right), \max \left(u_{i-\frac{1}{2}}, u_{i}, u_{i+\frac{1}{2}}\right)\right]$.

Then

$$
\sum_{i \in \mathbb{Z}}\left|\widehat{u}_{i+1}-\widehat{u}_{i}\right| \leq \sum_{i \in \mathbb{Z}}\left|u_{i+1}-u_{i}\right| .
$$

Proof. Let us first remark that the property

$$
\forall s_{1}, s_{2}, s_{3}, s_{4} \in \mathbb{R}, s_{1} \in\left[\min \left(s_{2}, s_{3}, s_{4}\right), \max \left(s_{2}, s_{3}, s_{4}\right)\right] \Rightarrow\left(\left|s_{3}-s_{4}\right|+\left|s_{3}-s_{2}\right| \geq\left|s_{1}-s_{4}\right|+\left|s_{1}-s_{2}\right|\right)
$$

can easily be shown by considering the different cases. We thus get that

$$
\left|u_{i}-u_{i-\frac{1}{2}}\right|+\left|u_{i}-u_{i+\frac{1}{2}}\right| \geq\left|\widehat{u}_{i}-u_{i-\frac{1}{2}}\right|+\left|\widehat{u}_{i}-u_{i+\frac{1}{2}}\right|, \forall i \in \mathbb{Z} .
$$

Using that, for all $i \in \mathbb{Z}$, we have $\left|u_{i}-u_{i+1}\right|=\left|u_{i}-u_{i+\frac{1}{2}}\right|+\left|u_{i+\frac{1}{2}}-u_{i+1}\right|$, since $u_{i+\frac{1}{2}} \in \overline{\mathrm{I}}\left(u_{i}, u_{i+1}\right)$, and $\left|\widehat{u}_{i}-\widehat{u}_{i+1}\right| \leq\left|u_{i+\frac{1}{2}}-\widehat{u}_{i}\right|+\left|\widehat{u}_{i+1}-u_{i+\frac{1}{2}}\right|$, we conclude the proof of the lemma. 
Theorem 5.5 (A variant of Ascoli's theorem) Let $E$ be a Banach space, let $\left(u_{n}\right)_{n \in \mathbb{N}}$ be a sequence of functions from $\mathbb{R}_{+} \rightarrow E$ such that, for all $t \in \mathbb{R}_{+}$, there exists a relatively compact subset $A(t)$ of $E$ with $u_{n}(t) \in A(t)$ for all $n \in \mathbb{N}$. We assume that there exists a sequence $\left(\delta t_{n}\right)_{n \in \mathbb{N}}$ of non negative reals which converges to 0 , and that there exists $C>0$ with

$$
\left\|u_{n}\left(t_{2}\right)-u_{n}\left(t_{1}\right)\right\|_{E} \leq C\left(\left|t_{2}-t_{1}\right|+\delta t_{n}\right), \forall n \in \mathbb{N}, \forall t_{1}, t_{2} \in \mathbb{R}_{+} .
$$

Then there exists $u \in \operatorname{Lip}\left(\mathbb{R}_{+} ; E\right)$ and a subsequence of $\left(u_{n}, \delta t_{n}\right)_{n \in \mathbb{N}}$, again denoted $\left(u_{n}, \delta t_{n}\right)_{n \in \mathbb{N}}$, such that

$$
\forall T \in \mathbb{R}_{+}, \lim _{n \rightarrow \infty} \sup _{t \in[0, T]}\left\|u_{n}(t)-u(t)\right\|_{E}=0 .
$$

Proof. The proof follows that of Ascoli's theorem. Let $\left(t_{n}\right)_{n \in \mathbb{N}}$ be a dense sequence in $\mathbb{R}_{+}$. One extracts from the sequence $\left(u_{n}, \delta t_{n}\right)_{n \in \mathbb{N}}$, thanks to the diagonal process, a subsequence such that $\left(u_{n}\left(t_{k}\right)\right)_{n \in \mathbb{N}}$ converges for all $k \in \mathbb{N}$.

Then the property (95) allows to show that, for all $t \in \mathbb{R}_{+},\left(u_{n}(t)\right)_{n \in \mathbb{N}}$ is a Cauchy sequence. Indeed, for $\varepsilon>0$, one first chooses $k \in \mathbb{N}$ such that $\left|t-t_{k}\right| \leq \varepsilon$, then $n_{0} \in \mathbb{N}$ such that $\delta t_{n} \leq \varepsilon$ for all $n \geq n_{0}$, and $\left\|u_{n}\left(t_{k}\right)-u_{p}\left(t_{k}\right)\right\|_{E} \leq \varepsilon$ for all $n, p \geq n_{0}$. The inequality $\left\|u_{n}(t)-u_{p}(t)\right\|_{E} \leq\left\|u_{n}(t)-u_{n}\left(t_{k}\right)\right\|_{E}+$ $\left\|u_{n}\left(t_{k}\right)-u_{p}\left(t_{k}\right)\right\|_{E}+\left\|u_{p}\left(t_{k}\right)-u_{p}(t)\right\|_{E} \leq(1+4 C) \varepsilon$ for all $n, p \geq n_{0}$ follows.

One then defines, for all $t \in \mathbb{R}_{+}, u(t)$ as the limit of $\left(u_{n}(t)\right)_{n \in \mathbb{N}}$. Passing to the limit $n \rightarrow \infty$ in (95) provides

$$
\left\|u\left(t_{2}\right)-u\left(t_{1}\right)\right\|_{E} \leq C\left|t_{2}-t_{1}\right|, \forall t_{1}, t_{2} \in \mathbb{R}_{+},
$$

which shows that $u \in \operatorname{Lip}\left(\mathbb{R}_{+} ; E\right)$. Then (96) is again an easy consequence of (95). Indeed, let $T \geq 0$ and $\varepsilon>0$ be given. Since, for all $k=0, \ldots,\lfloor T / \varepsilon\rfloor$ (where $\lfloor x\rfloor$ denotes the greater integer lower of equal to $x$ ), the sequence $\left(u_{n}(k \varepsilon)\right)_{n \in \mathbb{N}}$ converges to $u(k \varepsilon)$, let $n_{0} \in \mathbb{N}$ be such that $\left\|u_{n}(k \varepsilon)-u(k \varepsilon)\right\|_{E} \leq \varepsilon$ for all $k=0, \ldots,\lfloor T / \varepsilon\rfloor$ and all $n \geq n_{0}$, and such that $\delta t_{n} \leq \varepsilon$ for all $n \geq n_{0}$. Then, for all $t \in[0, T]$ and $n \geq n_{0}$, letting $k=\lfloor t / \varepsilon\rfloor$, we get using (97) and (95), $\left\|u(t)-u_{n}(t)\right\|_{E} \leq\|u(t)-u(k \varepsilon)\|_{E}+\left\|u(k \varepsilon)-u_{n}(k \varepsilon)\right\|_{E}+$ $\left\|u_{n}(k \varepsilon)-u_{n}(t)\right\|_{E} \leq(1+3 C) \varepsilon$, which concludes the proof of $(96)$.

\section{References}

[1] K. Aziz and A. Settari. Petroleum reservoir simulation. Applied Science Publishers, 1979.

[2] S. Bachu, M.A. Celia, and J.M. Nordbotten. Injection and Storage of $\mathrm{CO}_{2}$ in Deep Saline Aquifers: Analytical Solution for $\mathrm{CO}_{2}$ Plume Evolution During Injection. Transport in Porous Media, 58(3):339 - 360, March 2005.

[3] Y. Brenier and J. Jaffré. Upstream differencing for multiphase flow in reservoir simulation. SIAM J. Numer. Anal., 28(3):685-696, 1991.

[4] E. Brosse, Y. Le Gallo, and C.C. Magnier. Long-Term Mineral Trapping of CO2 in Aquifers and Reservoirs: Integration of Thermodynamics and Kinetics in Reservoir Engineering and Geological Simulations. Houston, March 2002. proceedings of AAPG Conference.

[5] J. Bruining, D. Marchesin, and C. J. Van Duijn. Steam injection into water-saturated porous rock. Comput. Appl. Math., 22(3):359-395, 2003.

[6] R. Eymard, T. Gallouët, and R. Herbin. Finite volume methods. In Ciarlet, P. G. (ed.) et al., Handbook of numerical analysis. Vol. \%: Solution of equations in $\mathbb{R}^{\ltimes}$ (Part 3). Techniques of scientific computing (Part 3). Amsterdam: North-Holland/ Elsevier. 713-1020 . 2000.

[7] R. Eymard, T. Gallouët, and J. Vovelle. Limit boundary conditions for finite volume approximations of some physical problems. J. Comput. Appl. Math., 2003. 
[8] Y. Le Gallo, P. Couillens, and T. Manaï. CO2 Sequestration in Depleted Oil or Gas Reservoirs. Kuala Lumpur, Malaysia, March 2002. SPE International Conference on Health, Safety and Environment in Oil and Gas Exploration and Production.

[9] E. Godlewski and P.A. Raviart. Hyperbolic systems of conservation laws. New York, 1996.

[10] S.K. Godunov. A difference method for numerical calculation of discontinuous solutions of the equations of hydrodynamics. Mat. Sb. (N.S.), 47:271-306, 1959.

[11] A. Holstad. A mathematical and numerical model for reactive fluid flow systems. Computational Geosciences, 4(2):103-139, Jun 2000.

[12] S.N. Krushkov. First order quasilinear equations in several independent variables. Math. USSR, Sb., 10:217-243, 1970.

[13] V. Lagneau, A. Pipart, and H. Catalette. Reactive Transport and Long Term Behaviour of $\mathrm{CO}_{2}$ Sequestration in Saline Aquifers. Oil and Gas Science and Technology, 60(2):231-247, 2005.

[14] T.P. Liu. The Riemann problem for general $2 \times 2$ conservation laws. Trans. Am. Math. Soc., 199:89-112, 1974.

[15] T.P. Liu. The Riemann problem for general systems of conservation laws. J. Differ. Equations, 18:218-234, 1975.

[16] T.P. Liu. The entropy condition and the admissibility of shocks. Arch. Rat. Mech. Anal., pages 78-88, 1976.

[17] L. Nghiem, P. Sammon, and J. Grabenstetter. Modeling CO2 Storage in Aquifers with a FullyCoupled Geochemical EOS Compositional Simulator. SPE/DOE Symposium on Improved Oil Recovery, 17-21 April, Tulsa, Oklahoma, 2004.

[18] O.A. Olejnik. Discontinuous solutions of non-linear differential equations. Translated by George Biriuk. Am. Math. Soc., Transl., II. Ser., 26:95-172, 1957.

[19] F. Otto. Initial-boundary value problem for a scalar conservation law. C. R. Acad. Sci., Paris, Sér., I 322(8):729-734, 1996.

[20] D. Serre. Systèmes de lois de conservation. I: Hyperbolicité, entropies, ondes de choc. (Systems of conservation laws. I: Hyperbolicity, entropy, shock waves). Fondations. Paris: Diderot Editeur. xii, 298 p. FF $180.00,1996$.

Robert EYMARD, Université de Marne-la-Vallée, 5, boulevard Descartes Champs-sur-Marne F-77454 Marne-la-Vallée CEDEX 2, France

Élodie TILLIER, 1 \&3 4, avenue de Bois-Préau F-92852 Rueil-Malmaison CEDEX, France 

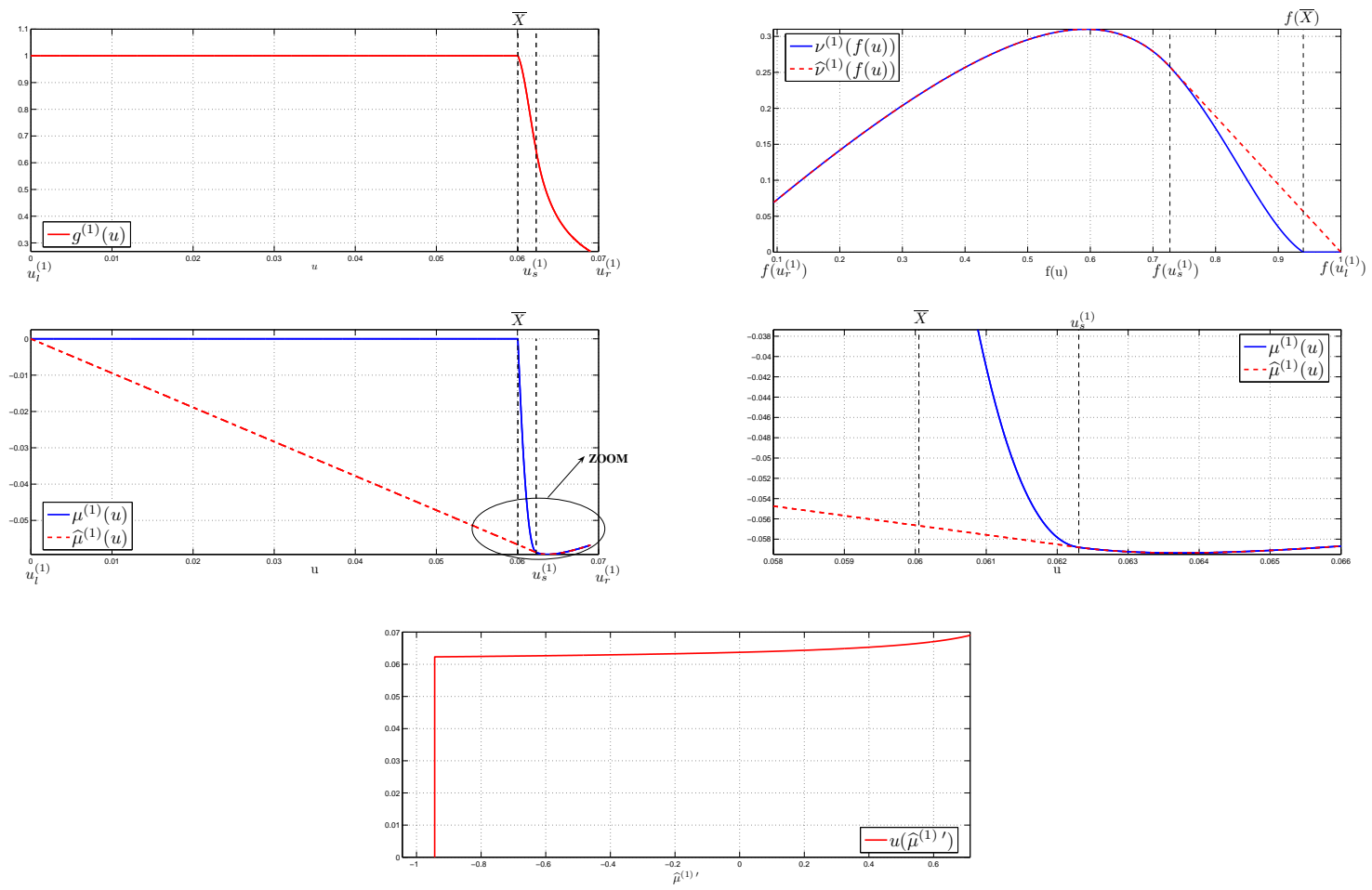

Figure 1: Functions $g^{(1)}$ (top left), $\nu^{(1)}$ (top right) with $\widehat{\nu}^{(1)}$ (dashed line), $\mu^{(1)}$ (middle left) with $\widehat{\mu}^{(1)}$ (dashed line) and an enlargement of this function (middle right). and $u^{(1)}$ as a function of $\left(\widehat{\mu}^{(1)}\right)^{\prime}$, which gives the profile of the solution for all time $t$ (bottom)
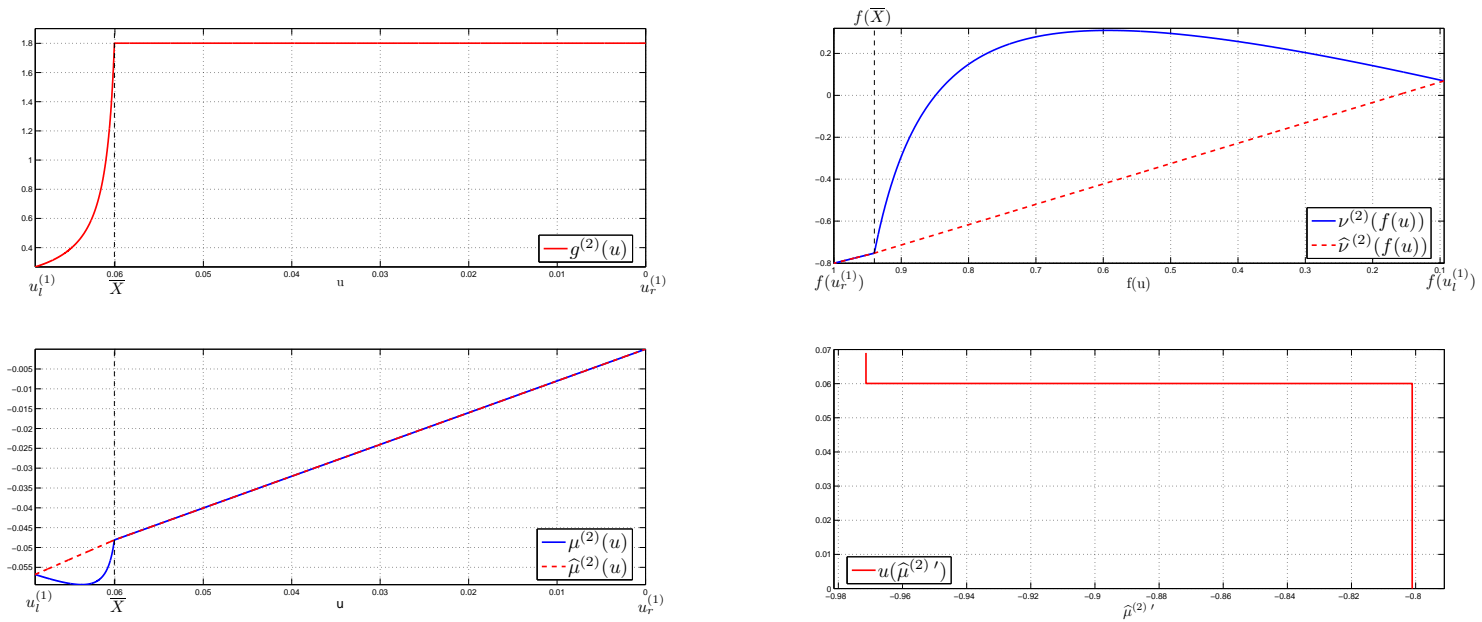

Figure 2: Functions $g^{(2)}$ (top left), $\nu^{(2)}$ (top right) with $\widehat{\nu}^{(2)}$ (dashed line), $\mu^{(2)}$ (middle left) with $\widehat{\mu}^{(2)}$ (dashed line) and $u^{(2)}$ as a function of $\left(\widehat{\mu}^{(2)}\right)^{\prime}$, which gives the profile of the solution for all time $t$ (bottom) 


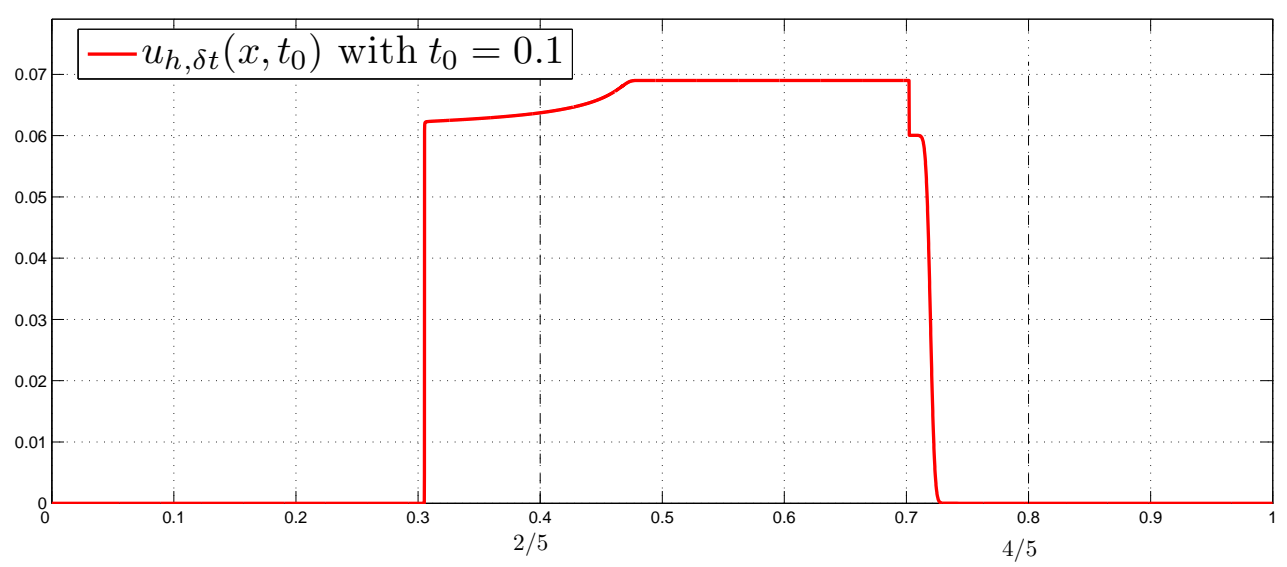

Figure 3: $u_{h, \delta t}\left(x, t_{0}\right)$ with $t_{0}=0.1$

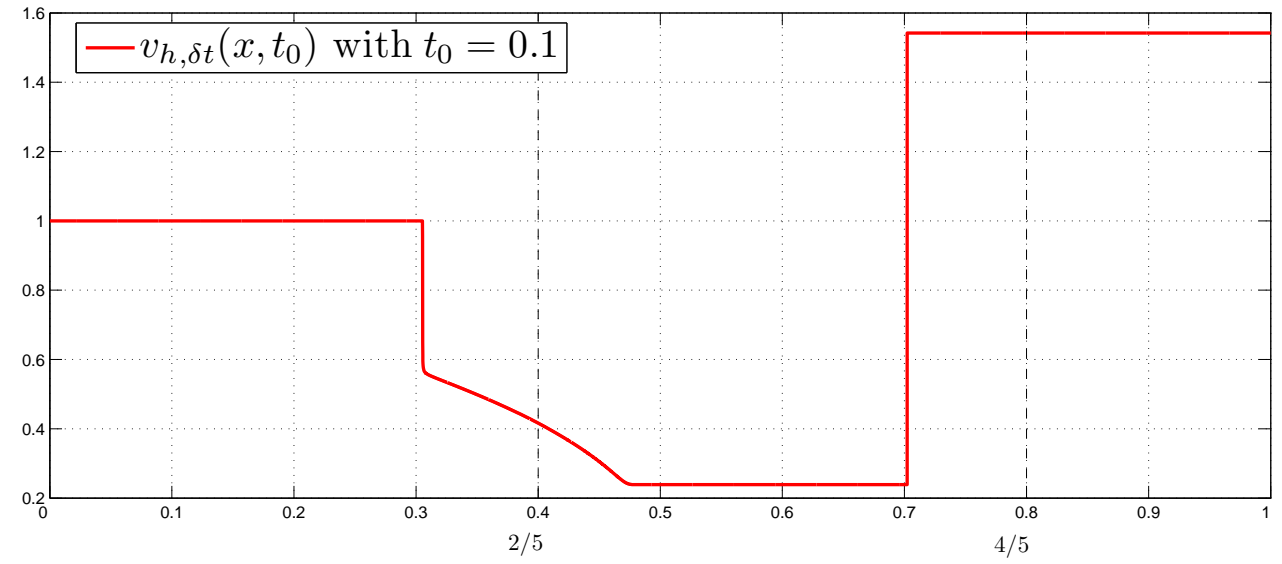

Figure 4: $v_{h, \delta t}\left(x, t_{0}\right)$ with $t_{0}=0.1$

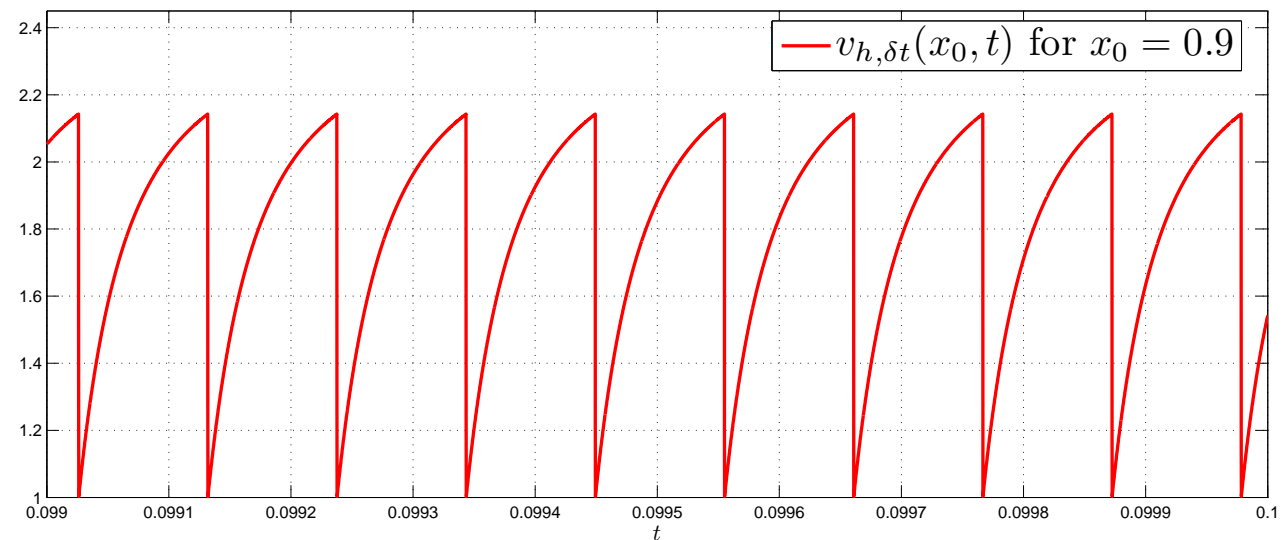

Figure 5: $v_{h, \delta t}\left(x_{0}, t\right)$ for $x_{0}=0.9$ 


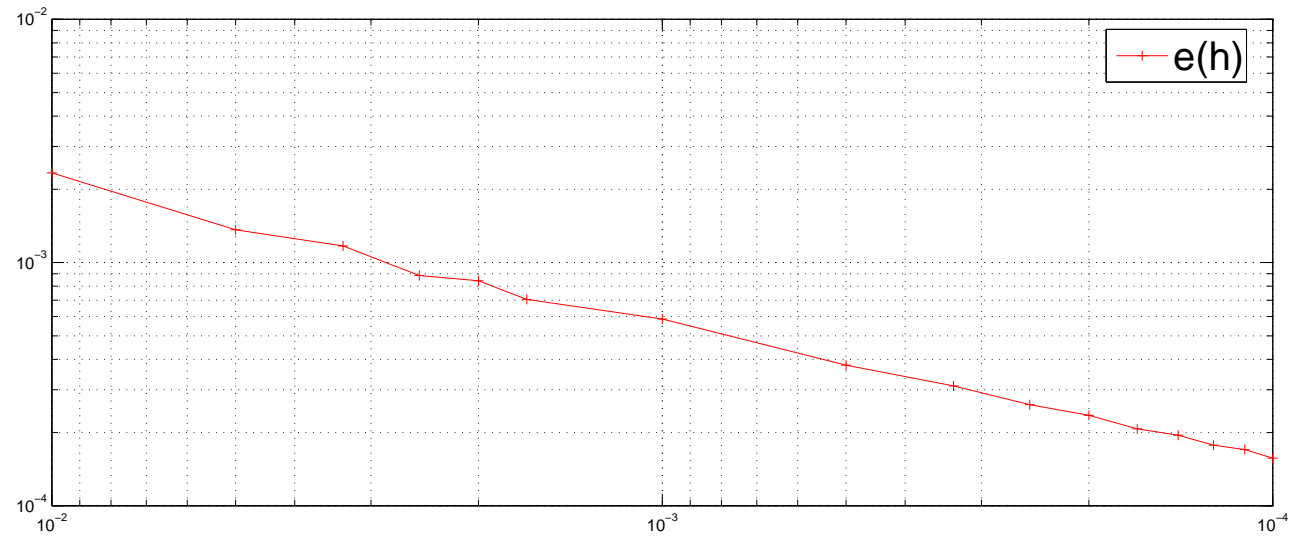

Figure 6: $e(h)$ 